\title{
Curves and envelopes that bound the spectrum of a matrix
}

\author{
Göran Bergqvist \\ Department of Mathematics, Linköping University, \\ SE-581 83 Linköping, Sweden \\ gober@mai.liu.se
}

\begin{abstract}
A generalization of the method developed by Adam, Psarrakos and Tsatsomeros to find inequalities for the eigenvalues of a complex matrix $A$ using knowledge of the largest eigenvalues of its Hermitian part $H(A)$ is presented. The numerical range or field of values of $A$ can be constructed as the intersection of half-planes determined by the largest eigenvalue of $H\left(e^{i \theta} A\right)$. Adam, Psarrakos and Tsatsomeros showed that using the two largest eigenvalues of $H(A)$, the eigenvalues of $A$ satisfy a cubic inequality and the envelope of such cubic curves defines a region in the complex plane smaller than the numerical range but still containing the spectrum of $A$. Here it is shown how using the three largest eigenvalues of $H(A)$ or more, one obtains new inequalities for the eigenvalues of $A$ and new envelope-type regions containing the spectrum of $A$.
\end{abstract}

Keywords: spectrum localization, eigenvalue inequalities, envelope, numerical range

AMS classification codes: 15A18, 15A42, 15A60, 65F15

\section{Introduction}

In this paper, we denote by $\mathcal{M}_{n, k}(\mathbb{C})$ and $\mathcal{M}_{n, k}(\mathbb{R})$ the spaces of complex and real $n \times k$ matrices respectively; $\mathcal{M}_{n}(\mathbb{C})$ and $\mathcal{M}_{n}(\mathbb{R})$ stand for $k=n$. The spectrum $\sigma(A)$ of a matrix $A \in \mathcal{M}_{n}(\mathbb{C})$ is known to be located in its numerical range or field of values $F(A)=\left\{\mathbf{x}^{*} A \mathbf{x} \in \mathbb{C} ; \mathbf{x} \in \mathbb{C}^{n},\|\mathbf{x}\|_{2}=\right.$ $1\}$. The spectrum of $A$ is also located to the left of the vertical line $\operatorname{Re}(z)=\delta_{1}$ in the complex plane, where $\delta_{1}$ is the largest eigenvalue of the Hermitian part $H(A)=\frac{1}{2}\left(A+A^{*}\right)$ of $A$. Here $A^{*}$ denotes the Hermitian conjugate of $A$. Also, $S(A)=\frac{1}{2}\left(A-A^{*}\right)$ denotes the skew-Hermitian part of $A$, so $A=H(A)+S(A)$.

Clearly $F(A)=e^{-i \theta} F\left(e^{i \theta} A\right)$ for any $\theta \in\left[0,2 \pi\left[\right.\right.$, so $e^{i \theta} F(A)$ is located to the left of the vertical line $\operatorname{Re}(z)=\lambda_{\max }\left(H\left(e^{i \theta} A\right)\right)$, and rotating this line by $e^{-i \theta}$ we get a new line that bounds $\sigma(A)$. In fact, [2, 3], $F(A)$ is obtained exactly as the connected, compact and convex region defined by the envelope of all such lines. In the first subfigure of Figure 1 we have illustrated this for the Toeplitz matrix

$$
A=\left(\begin{array}{llll}
1 & 1 & 0 & i \\
2 & 1 & 1 & 0 \\
3 & 2 & 1 & 1 \\
4 & 3 & 2 & 1
\end{array}\right)
$$




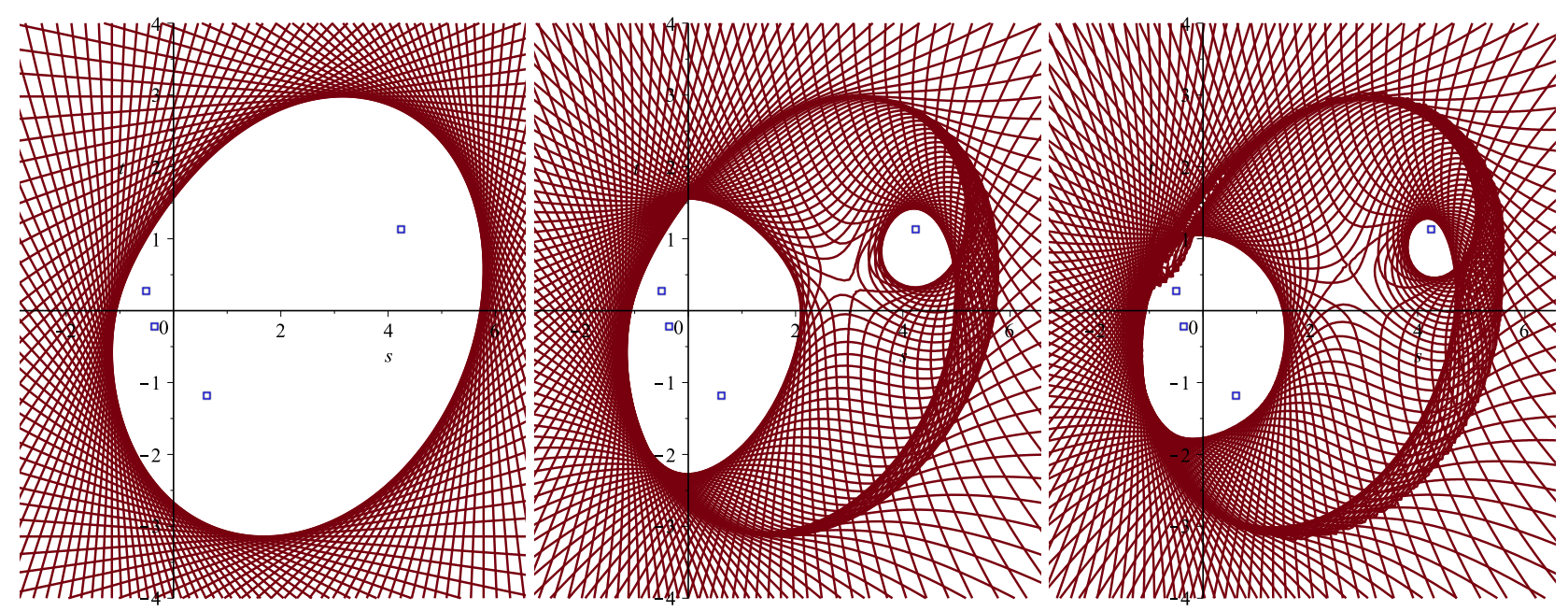

Figure 1: The numerical range $F(A)$ (left), the regions $\mathcal{E}_{1}(A)$ (middle) and $\mathcal{E}_{2}(A)$ (right) of $A$ in (1).

by plotting these lines for $\theta=2 \pi m / 120, m=0, \ldots, 119$. The eigenvalues of $A$ are marked by small boxes in the figure.

In [1] Adam and Tsatsomeros showed how one can use the two largest eigenvalues $\delta_{1}$ and $\delta_{2}$ of $H(A)$ and the eigenvector $\mathbf{u}_{1}$ of $H(A)$ corresponding to $\delta_{1}$, to obtain an improved inequality for $\sigma(A)$. Let $\alpha=\operatorname{Im}\left(\mathbf{u}_{1}^{*} S(A) \mathbf{u}_{1}\right)$ and $K_{1}=\left\|S(A) \mathbf{u}_{1}\right\|_{2}^{2}-\alpha^{2} \geq 0$. Then they proved that any $\lambda \in \sigma(A)$ satisfies

$$
\left|\lambda-\left(\delta_{1}+i \alpha\right)\right|^{2}\left(\operatorname{Re}(\lambda)-\delta_{2}\right) \leq K_{1}\left(\delta_{1}-\operatorname{Re}(\lambda)\right) .
$$

With equality in (2) we have a curve $\Gamma_{1}(A)$ that bounds $\sigma(A)$ and is of degree 3 in the real and imaginary parts of $\lambda$. Applied to $H\left(e^{i \theta} A\right)$ one can repeat the argument above and obtain rotated cubic curves that bound $\sigma(A)$. The envelope of such curves was studied extensively by Psarrakos and Tsatsomeros [5, 6] and they showed that it bounds a region $\mathcal{E}_{1}(A)$ that contains $\sigma(A)$ and is compact, but which is not always convex or connected. In the second subfigure of Figure 1 we show their image [5] of $\mathcal{E}_{1}(A)$ for the Toeplitz matrix $A$ in (11). Again we used 120 curves, with $\theta=2 \pi m / 120, m=0, \ldots, 119$, for the plot.

The aim of this paper is to generalize the results of Adam, Psarrakos and Tsatsomeros by using the $k$ largest eigenvalues of $H(A)$ to obtain new curves $\Gamma_{k}(A)$ that bound $\sigma(A)$. As a preview of our results, we show in the third subfigure of Figure 1 the region $\mathcal{E}_{2}(A)$ obtained from the envelope of curves when the three largest eigenvalues of $H(A)$ are utilized for the Toeplitz matrix $A$ in (1). Also here 120 curves are used to construct the figure.

In Section 2 we derive the main inequality for the eigenvalues of a matrix, with respect to the largest eigenvalues and corresponding eigenvectors of its Hermitian part. We also state a more explicit formulation for the case of three known eigenvalues, and present some illustrations and analyze properties of the curves that bound the spectrum. In Section 3 we analyze explicitly some cases where the curves have special properties. Then, in Section 4, we demonstrate how an envelope of such curves encloses a region which is inside the numerical range and contains the spectrum. We compare this region with the region obtained for the case of two known eigenvalues presented by Psarrakos and Tsatsomeros. 


\section{Eigenvalue inequalities and spectrum bounding curves}

Let $A \in \mathcal{M}_{n}(\mathbb{C})$ and let $\delta_{1} \geq \cdots \geq \delta_{n}$ be the eigenvalues of $H(A)$ with $\mathbf{u}_{1}, \ldots, \mathbf{u}_{n}$ the corresponding normalized orthogonal eigenvectors. Consider the unitary matrix $U \in \mathcal{M}_{n}(\mathbb{C})$ with columns $\mathbf{u}_{j}$, the diagonal matrix $\Delta=\operatorname{diag}\left(\delta_{1}, \ldots, \delta_{n}\right) \in \mathcal{M}_{n}(\mathbb{R})$ with diagonal elements $\delta_{1}, \ldots, \delta_{n}$, and formulate the Hermitian part of $A$ as

$$
H(A)=U \Delta U^{*} \Leftrightarrow U^{*} H(A) U=\Delta=\left(\begin{array}{cc}
\Delta_{k} & 0 \\
0 & \tilde{\Delta}_{k}
\end{array}\right)
$$

where $\Delta_{k}=\operatorname{diag}\left(\delta_{1}, \ldots, \delta_{k}\right) \in \mathcal{M}_{k}(\mathbb{R})$ and $\tilde{\Delta}_{k}=\operatorname{diag}\left(\delta_{k+1}, \ldots, \delta_{n}\right) \in \mathcal{M}_{n-k}(\mathbb{R})$. Define the skew-Hermitian matrix

$$
Y=U^{*} S(A) U=\left(\begin{array}{cc}
Y_{k} & -V_{k}^{*} \\
V_{k} & \tilde{Y}_{k}
\end{array}\right)
$$

where $Y_{k} \in \mathcal{M}_{k}(\mathbb{C})$ and $\tilde{Y}_{k} \in \mathcal{M}_{n-k}(\mathbb{C})$ are skew-Hermitian, and $V_{k} \in \mathcal{M}_{n-k, k}(\mathbb{C})$. Now, let the upper principal $k \times k$ submatrix of $U^{*}\left(A-\lambda I_{n}\right) U$ be

$$
W_{k}=\Delta_{k}+Y_{k}-\lambda I_{k},
$$

where $\lambda=s+i t$ is an eigenvalue of $A$. Obviously, combining (3) - (5) for the Hermitian part of $W_{k}$, we have $H\left(W_{k}\right)=\frac{1}{2}\left(W_{k}+W_{k}^{*}\right)=\Delta_{k}-s I_{k}$.

Lemma 1. If $\lambda \in \sigma(A) \backslash \sigma\left(\Delta_{k}+Y_{k}\right)$, then $H\left(W_{k}^{-1}\right)$ is not negative definite.

Proof. $W_{k}^{-1}$ exists since $\lambda \notin \sigma\left(\Delta_{k}+Y_{k}\right)$. Since $s \leq \delta_{1}$ for $\lambda \in \sigma(A), H\left(W_{k}\right)=\operatorname{diag}\left(\delta_{1}-\right.$ $\left.s, \ldots, \delta_{k}-s\right)$ has at least one non-negative eigenvalue $\delta_{1}-s$ and is therefore not negative definite, which is equivalent to $H\left(W_{k}^{-1}\right)$ not being negative definite [4].

Recall that the adjugate $\operatorname{adj}(A) \in \mathcal{M}_{n}(\mathbb{C})$ of $A \in \mathcal{M}_{n}(\mathbb{C})$ is the matrix whose elements are minors of $A$, and satisfies $\operatorname{adj}(A) A=(\operatorname{det} A) I_{n}$. The following is the main result of the paper.

Theorem 2. Let $A \in \mathcal{M}_{n}(\mathbb{C})$ and $\lambda$ be an eigenvalue of $A$. Let $\delta_{1} \geq \cdots \geq \delta_{n}$ be the eigenvalues of the Hermitian part of $A$. Then

$$
\left|\operatorname{det} W_{k}\right|^{2}\left(\operatorname{Re}(\lambda)-\delta_{k+1}\right) \leq\left(\sigma_{1}\left(V_{k}\right)\right)^{2} \lambda_{\max }\left(H\left(\operatorname{det} W_{k} \operatorname{adj}\left(W_{k}^{*}\right)\right)\right),
$$

where $V_{k}$ and $W_{k}$ are defined in (4) and (5) respectively, adj $\left(W_{k}^{*}\right)$ is the adjugate of $W_{k}^{*}$, $\lambda_{\text {max }}\left(H\left(\operatorname{det} W_{k} \operatorname{adj}\left(W_{k}^{*}\right)\right)\right) \geq 0$ is the largest eigenvalue of the Hermitian part of $\operatorname{det} W_{k} \operatorname{adj}\left(W_{k}^{*}\right)$, and $\sigma_{1}\left(V_{k}\right)$ is the largest singular value of $V_{k}$.

Proof. We adopt the ideas from the proof of the case $k=1$ presented by Adam and Tsatsomeros [1. Let $\lambda=s+i t$ be an eigenvalue of $A$. This means that $A-\lambda I_{n}$ and

$$
U^{*}\left(A-\lambda I_{n}\right) U=\left(\begin{array}{cc}
W_{k} & -V_{k}^{*} \\
V_{k} & \tilde{\Delta}_{k}+\tilde{Y}_{k}-\lambda I_{n-k}
\end{array}\right),
$$

are singular. If $W_{k}$ is singular, then $\operatorname{det} W_{k}=0$ and the statement of the theorem is trivial. For the case that $W_{k}$ is nonsingular, the Schur complement $\tilde{W}_{k}=\tilde{\Delta}_{k}+\tilde{Y}_{k}-\lambda I_{n-k}+V_{k} W_{k}^{-1} V_{k}^{*}$ of $W_{k}$ must be singular, since $0=\operatorname{det}\left(U^{*}\left(A-\lambda I_{n}\right) U\right)=\operatorname{det} W_{k} \operatorname{det} \tilde{W}_{k}$. Hence $0 \in \sigma\left(\tilde{W}_{k}\right) \subset$ 
$F\left(\tilde{W}_{k}\right)$, which implies $0 \in \operatorname{Re}\left(F\left(\tilde{W}_{k}\right)\right)=F\left(H\left(\tilde{W}_{k}\right)\right)$, 2]. Consequently there exists a unit vector $\mathbf{x} \in \mathbb{C}^{n-k}$ such that

$$
\mathbf{x}^{*} H\left(\tilde{W}_{k}\right) \mathbf{x}=0
$$

Since

$H\left(\tilde{W}_{k}\right)=\frac{1}{2}\left(\tilde{W}_{k}+\tilde{W}_{k}^{*}\right)=\tilde{\Delta}_{k}-s I_{n-k}+\frac{1}{2} V_{k}\left(W_{k}^{-1}+\left(W_{k}^{-1}\right)^{*}\right) V_{k}^{*}=\tilde{\Delta}_{k}-s I_{n-k}+V_{k} H\left(W_{k}^{-1}\right) V_{k}^{*}$, from (7) we get

$$
0=\mathbf{x}^{*} \tilde{\Delta}_{k} \mathbf{x}-s \mathbf{x}^{*} I_{n-k} \mathbf{x}+\mathbf{x}^{*} V_{k} H\left(W_{k}^{-1}\right) V_{k}^{*} \mathbf{x}=\mathbf{x}^{*} \tilde{\Delta}_{k} \mathbf{x}-s+\left(V_{k}^{*} \mathbf{x}\right)^{*} H\left(W_{k}^{-1}\right)\left(V_{k}^{*} \mathbf{x}\right) .
$$

Further, using the unit vector $\mathbf{x}$, the largest eigenvalues $\delta_{k+1}$ and $\lambda_{\max }\left(H\left(W_{k}^{-1}\right)\right)$ of the Hermitian matrices $\tilde{\Delta}_{k}$ and $H\left(W_{k}^{-1}\right)$, respectively, and the largest singular value $\sigma_{1}\left(V_{k}^{*}\right)$ of $V_{k}^{*}$, we obtain

$$
\begin{gathered}
\mathbf{x}^{*} \tilde{\Delta}_{k} \mathbf{x} \leq \delta_{k+1}\|\mathbf{x}\|_{2}^{2}=\delta_{k+1} \\
\left(V_{k}^{*} \mathbf{x}\right)^{*} H\left(W_{k}^{-1}\right)\left(V_{k}^{*} \mathbf{x}\right) \leq \lambda_{\max }\left(H\left(W_{k}^{-1}\right)\right)\left\|V_{k}^{*} \mathbf{x}\right\|_{2}^{2},
\end{gathered}
$$

and

$$
\left\|V_{k}^{*} \mathbf{x}\right\|_{2}^{2} \leq\left(\sigma_{1}\left(V_{k}^{*}\right)\right)^{2}\|\mathbf{x}\|_{2}^{2}=\left(\sigma_{1}\left(V_{k}^{*}\right)\right)^{2}=\left(\sigma_{1}\left(V_{k}\right)\right)^{2} .
$$

Also, according to Lemma 1 for the largest eigenvalue of $H\left(W_{k}^{-1}\right)$ we have $\lambda_{\max }\left(H\left(W_{k}^{-1}\right)\right) \geq 0$, which together with (11) gives

$$
\lambda_{\max }\left(H\left(W_{k}^{-1}\right)\right)\left\|V_{k}^{*} \mathbf{x}\right\|_{2}^{2} \leq \lambda_{\max }\left(H\left(W_{k}^{-1}\right)\right)\left(\sigma_{1}\left(V_{k}\right)\right)^{2} .
$$

Using the inequalities (9), (10) and $(12)$ in $(8)$ we get

$$
0 \leq \delta_{k+1}-s+\lambda_{\max }\left(H\left(W_{k}^{-1}\right)\right)\left(\sigma_{1}\left(V_{k}\right)\right)^{2} .
$$

The inequality $(13)$ is our main result in the case of a nonsingular $W_{k}$. In order to formulate (6), which is valid also for a singular $W_{k}$, notice that for $H\left(W_{k}^{-1}\right)$ we have

$$
H\left(W_{k}^{-1}\right)=\frac{1}{2}\left(W_{k}^{-1}+\left(W_{k}^{-1}\right)^{*}\right)=\frac{1}{2}\left(\frac{1}{\operatorname{det} W_{k}} \operatorname{adj}\left(W_{k}\right)+\frac{1}{\overline{\operatorname{det} W_{k}}} \operatorname{adj}\left(W_{k}^{*}\right)\right)=\frac{M_{k}}{\left|\operatorname{det} W_{k}\right|^{2}},
$$

where

$$
M_{k}=\frac{1}{2}\left(\overline{\operatorname{det} W_{k}} \operatorname{adj}\left(W_{k}\right)+\operatorname{det} W_{k} \operatorname{adj}\left(W_{k}^{*}\right)\right)=H\left(\operatorname{det} W_{k} \operatorname{adj}\left(W_{k}^{*}\right)\right) .
$$

Hence,

$$
\lambda_{\max }\left(H\left(W_{k}^{-1}\right)\right)=\frac{1}{\left|\operatorname{det} W_{k}\right|^{2}} \lambda_{\max }\left(M_{k}\right) .
$$

Multiplying (13) by $\left|\operatorname{det} W_{k}\right|^{2}$ and using (15), the inequality (6) follows.

Note that $\lambda_{\max }\left(M_{k}\right)$ is a non-negative function of $s$ and $t$ while $\sigma_{1}\left(V_{k}\right)$ is a constant.

For $k=1, Y_{1}=i \alpha$ where $\alpha=-i \mathbf{u}_{1}^{*} S(A) \mathbf{u}_{1} \in \mathbb{R}, V_{1}$ is a vector, and $W_{1}=\delta_{1}-s+i(\alpha-t)$ is a scalar. Interpreting the adjugate of an $1 \times 1$ matrix as 1 (to keep $\operatorname{adj}(A) A=(\operatorname{det} A) I_{n}$ that was used in the proof), using (5) and (14) we have $M_{1}=\delta_{1}-s$ and $\lambda_{\max }\left(M_{1}\right)=M_{1}$. Moreover, 
for the column $\mathbf{y}_{1}$ of $Y$ in (4) we have $\left\|S(A) \mathbf{u}_{1}\right\|_{2}^{2}=\mathbf{y}_{1}^{*} \mathbf{y}_{1}=\alpha^{2}+V_{1}^{*} V_{1} \Rightarrow K_{1}=\left(\sigma_{1}\left(V_{1}\right)\right)^{2} \equiv$ $V_{1}^{*} V_{1}=\left\|S(A) \mathbf{u}_{1}\right\|_{2}^{2}-\alpha^{2}$, whereby we obtain the result of Adam and Tsatsomeros [1]

$$
\left[\left(\delta_{1}-s\right)^{2}+(\alpha-t)^{2}\right]\left(s-\delta_{2}\right) \leq K_{1}\left(\delta_{1}-s\right) .
$$

We use the notation $\Gamma_{1}(A)$ for the cubic curve obtained from equality in 16 . More generally:

Definition 1. $\Gamma_{k}(A)$ is the curve $\left|\operatorname{det} W_{k}\right|^{2}\left(\operatorname{Re}(\lambda)-\delta_{k+1}\right)=\left(\sigma_{1}\left(V_{k}\right)\right)^{2} \lambda_{\max }\left(H\left(\operatorname{det} W_{k} \operatorname{adj}\left(W_{k}^{*}\right)\right)\right)$ obtained from having equality in (6) of Theorem 2 .

We use the variables $s$ and $t$ for the curve, where $s+i t=\lambda$ in the expression (5) for $W_{k}$.

For $k=2$ we now state an explicit inequality which gives an expression for the curve $\Gamma_{2}(A)$. In this case

$$
W_{2}=\Delta_{2}+Y_{2}-\lambda I_{2}=\left(\begin{array}{cc}
\delta_{1}-s+i(\alpha-t) & -\bar{\gamma} \\
\gamma & \delta_{2}-s+i(\beta-t)
\end{array}\right),
$$

where $i \alpha=\mathbf{u}_{1}^{*} S(A) \mathbf{u}_{1}, i \beta=\mathbf{u}_{2}^{*} S(A) \mathbf{u}_{2}, \gamma=\mathbf{u}_{2}^{*} S(A) \mathbf{u}_{1}$, and $V_{2} \in \mathcal{M}_{n-2,2}(\mathbb{C})$ is such that the first two columns of $Y$ in (4) are

$$
\mathcal{M}_{n, 2}(\mathbb{C}) \ni\left(\begin{array}{l}
Y_{2} \\
V_{2}
\end{array}\right)=\left(\begin{array}{cc}
i \alpha & -\bar{\gamma} \\
\gamma & i \beta \\
\mathbf{v}_{1} & \mathbf{v}_{2}
\end{array}\right)=U^{*} S(A)\left(\begin{array}{ll}
\mathbf{u}_{1} & \mathbf{u}_{2}
\end{array}\right)
$$

Proposition 3. Let $A \in \mathcal{M}_{n}(\mathbb{C})$ and $\lambda=s+i t$ be an eigenvalue of $A$. Let $\delta_{1} \geq \delta_{2} \geq \delta_{3}$ be the three largest eigenvalues of the Hermitian part of $A$, and let $\mathbf{u}_{1}$ and $\mathbf{u}_{2}$ be the corresponding normalized eigenvectors of $\delta_{1}$ and $\delta_{2}$. Then

$$
\begin{gathered}
{\left[\left(\left(\delta_{1}-s\right)\left(\delta_{2}-s\right)-(\alpha-t)(\beta-t)+|\gamma|^{2}\right)^{2}+\left(\left(\delta_{1}-s\right)(\beta-t)+\left(\delta_{2}-s\right)(\alpha-t)\right)^{2}\right]\left(s-\delta_{3}\right)} \\
\leq \frac{K_{2}}{2}\left[m_{1}(s, t)+m_{3}(s, t)+\sqrt{\left[m_{1}(s, t)-m_{3}(s, t)\right]^{2}+4\left|m_{2}(s, t)\right|^{2}}\right]
\end{gathered}
$$

where

$$
\begin{aligned}
& \alpha=-i \mathbf{u}_{1}^{*} S(A) \mathbf{u}_{1} \in \mathbb{R}, \quad \beta=-i \mathbf{u}_{2}^{*} S(A) \mathbf{u}_{2} \in \mathbb{R}, \quad \gamma=\mathbf{u}_{2}^{*} S(A) \mathbf{u}_{1} \in \mathbb{C} \\
& K_{2}=\frac{1}{2}\left[\left\|S(A) \mathbf{u}_{1}\right\|_{2}^{2}+|| S(A) \mathbf{u}_{2} \|_{2}^{2}-\alpha^{2}-\beta^{2}-2|\gamma|^{2}\right. \\
& \left.\quad+\sqrt{\left(\left\|S(A) \mathbf{u}_{1}\right\|_{2}^{2}-\left\|S(A) \mathbf{u}_{2}\right\|_{2}^{2}-\alpha^{2}+\beta^{2}\right)^{2}+4\left|\left(S(A) \mathbf{u}_{2}\right)^{*}\left(S(A) \mathbf{u}_{1}\right)+i \gamma(\alpha+\beta)\right|^{2}}\right] \\
& \\
& m_{1}(s, t)=\left(\delta_{1}-s\right)\left[\left(\delta_{2}-s\right)^{2}+(\beta-t)^{2}\right]+\left(\delta_{2}-s\right)|\gamma|^{2}, \\
& m_{2}(s, t)=i \gamma\left[\left(\delta_{1}-s\right)(\beta-t)+\left(\delta_{2}-s\right)(\alpha-t)\right], \\
& m_{3}(s, t)=\left(\delta_{2}-s\right)\left[\left(\delta_{1}-s\right)^{2}+(\alpha-t)^{2}\right]+\left(\delta_{1}-s\right)|\gamma|^{2} .
\end{aligned}
$$

Proof. By (17), we have

$$
\operatorname{det} W_{2}=\left(\delta_{1}-s\right)\left(\delta_{2}-s\right)-(\alpha-t)(\beta-t)+|\gamma|^{2}+i\left(\left(\delta_{1}-s\right)(\beta-t)+\left(\delta_{2}-s\right)(\alpha-t)\right),
$$


which gives

$$
\left|\operatorname{det} W_{2}\right|^{2}=\left(\left(\delta_{1}-s\right)\left(\delta_{2}-s\right)-(\alpha-t)(\beta-t)+|\gamma|^{2}\right)^{2}+\left(\left(\delta_{1}-s\right)(\beta-t)+\left(\delta_{2}-s\right)(\alpha-t)\right)^{2} .
$$

Furthermore

$\operatorname{adj}\left(W_{2}^{*}\right)=\operatorname{adj}\left(\begin{array}{cc}\delta_{1}-s-i(\alpha-t) & \bar{\gamma} \\ -\gamma & \delta_{2}-s-i(\beta-t)\end{array}\right)=\left(\begin{array}{cc}\delta_{2}-s-i(\beta-t) & -\bar{\gamma} \\ \gamma & \delta_{1}-s-i(\alpha-t)\end{array}\right)$,

and a straightforward calculation from (14) gives

$$
\begin{gathered}
M_{2}=\left(\begin{array}{cc}
m_{1}(s, t) & \bar{m}_{2}(s, t) \\
m_{2}(s, t) & m_{3}(s, t)
\end{array}\right)=H\left(\operatorname{det} W_{2} a \operatorname{adj}\left(W_{2}^{*}\right)\right)=\frac{1}{2}\left(\overline{\operatorname{det} W_{2}} \operatorname{adj}\left(W_{2}\right)+\operatorname{det} W_{2} \operatorname{adj}\left(W_{2}^{*}\right)\right)= \\
\left(\begin{array}{cc}
\left(\delta_{1}-s\right)\left[\left(\delta_{2}-s\right)^{2}+(\beta-t)^{2}\right]+\left(\delta_{2}-s\right)|\gamma|^{2} & -i \bar{\gamma}\left[\left(\delta_{1}-s\right)(\beta-t)+\left(\delta_{2}-s\right)(\alpha-t)\right] \\
i \gamma\left[\left(\delta_{1}-s\right)(\beta-t)+\left(\delta_{2}-s\right)(\alpha-t)\right] & \left(\delta_{2}-s\right)\left[\left(\delta_{1}-s\right)^{2}+(\alpha-t)^{2}\right]+\left(\delta_{1}-s\right)|\gamma|^{2}
\end{array}\right) .
\end{gathered}
$$

The largest eigenvalue of $M_{2}$ is

$$
\lambda_{\max }\left(M_{2}\right)=\frac{1}{2}\left(m_{1}+m_{3}+\sqrt{\left(m_{1}-m_{3}\right)^{2}+4\left|m_{2}\right|^{2}}\right) .
$$

To calculate $K_{2}=\left(\sigma_{1}\left(V_{2}\right)\right)^{2}=\lambda_{\max }\left(V_{2}^{*} V_{2}\right)$, use that $V_{2}=\left(\begin{array}{ll}\mathbf{v}_{1} & \mathbf{v}_{2}\end{array}\right)$ by $(18)$. Then $V_{2}^{*} V_{2}$ is the $2 \times 2$ Gram matrix

$$
V_{2}^{*} V_{2}=\left(\begin{array}{ll}
\mathbf{v}_{1}^{*} \mathbf{v}_{1} & \mathbf{v}_{1}^{*} \mathbf{v}_{2} \\
\mathbf{v}_{2}^{*} \mathbf{v}_{1} & \mathbf{v}_{2}^{*} \mathbf{v}_{2}
\end{array}\right)
$$

whose largest eigenvalue is

$$
K_{2}=\frac{1}{2}\left[\mathbf{v}_{1}^{*} \mathbf{v}_{1}+\mathbf{v}_{2}^{*} \mathbf{v}_{2}+\sqrt{\left(\mathbf{v}_{1}^{*} \mathbf{v}_{1}-\mathbf{v}_{2}^{*} \mathbf{v}_{2}\right)^{2}+4\left|\mathbf{v}_{2}^{*} \mathbf{v}_{1}\right|^{2}}\right] .
$$

Further, for the columns $\mathbf{y}_{j}$ of $Y$ in (4) we have

$$
\mathbf{y}_{j}^{*} \mathbf{y}_{k}=\left(U S(A) \mathbf{u}_{j}\right)^{*}\left(U S(A) \mathbf{u}_{k}\right)=\left(S(A) \mathbf{u}_{j}\right)^{*} U^{*} U\left(S(A) \mathbf{u}_{k}\right)=\left(S(A) \mathbf{u}_{j}\right)^{*}\left(S(A) \mathbf{u}_{k}\right) .
$$

Thus, combining (18) with the above equalities we get

$$
\alpha^{2}+|\gamma|^{2}+\mathbf{v}_{1}^{*} \mathbf{v}_{1}=\mathbf{y}_{1}^{*} \mathbf{y}_{1}=\left\|S(A) \mathbf{u}_{1}\right\|_{2}^{2},|\gamma|^{2}+\beta^{2}+\mathbf{v}_{2}^{*} \mathbf{v}_{2}=\mathbf{y}_{2}^{*} \mathbf{y}_{2}=\left\|S(A) \mathbf{u}_{2}\right\|_{2}^{2},
$$

and

$$
-i \gamma(\alpha+\beta)+\mathbf{v}_{2}^{*} \mathbf{v}_{1}=\mathbf{y}_{2}^{*} \mathbf{y}_{1}=\left(S(A) \mathbf{u}_{2}\right)^{*}\left(S(A) \mathbf{u}_{1}\right) .
$$

The substitution of these relations in $(23)$ yields

$$
\begin{aligned}
& K_{2}=\frac{1}{2}\left[\left\|S(A) \mathbf{u}_{1}\right\|_{2}^{2}+\left\|S(A) \mathbf{u}_{2}\right\|_{2}^{2}-\alpha^{2}-\beta^{2}-2|\gamma|^{2}\right. \\
& \left.\quad+\sqrt{\left(\left\|S(A) \mathbf{u}_{1}\right\|_{2}^{2}-\left\|S(A) \mathbf{u}_{2}\right\|_{2}^{2}-\alpha^{2}+\beta^{2}\right)^{2}+4\left|\left(S(A) \mathbf{u}_{2}\right)^{*}\left(S(A) \mathbf{u}_{1}\right)+i \gamma(\alpha+\beta)\right|^{2}}\right]
\end{aligned}
$$

Combining the equations (20), (21), (22), (24) and (6), the inequality in $(19)$ is derived for $k=2$. 
We now state some properties of the curves $\Gamma_{k}(A)$ of Definition 1 , which for $k=1$ have been proved in [5].

Proposition 4. For the curves $\Gamma_{k}(A)$ we have:

(i) $\Gamma_{k}\left(\tilde{U}^{*} A \tilde{U}\right)=\Gamma_{k}(A)$ if $\tilde{U}$ is unitary

(ii) $\Gamma_{k}\left(A^{T}\right)=\Gamma_{k}(A)$

(iii) $\Gamma_{k}\left(A^{*}\right)=\overline{\Gamma_{k}(A)}$

(iv) $\Gamma_{k}\left(r A+b I_{n}\right)=r \Gamma_{k}(A)+b$ if $0<r \in \mathbb{R}$ and $b \in \mathbb{C}$

Proof. ( $i)$ With $\tilde{A}=\tilde{U}^{*} A \tilde{U}$ we get $H(\tilde{A})=\tilde{U}^{*} H(A) \tilde{U}, S(\tilde{A})=\tilde{U}^{*} S(A) \tilde{U}$. Using the notation of (3), (4), (5) and Theorem 2, we have that $H(\tilde{A})$ is diagonalized by $\tilde{U}^{*} U:\left(\tilde{U}^{*} U\right)^{*} H(\tilde{A})\left(\tilde{U}^{*} U\right)=$ $U^{*} H(A) U=\Delta$, so $\Delta$ is invariant. Then $\left(\tilde{U}^{*} U\right)^{*} S(\tilde{A})\left(\tilde{U}^{*} U\right)=U^{*} S(A) U=Y$ is also invariant, which implies that all quantities $W_{k}, V_{k}$, and $\delta_{k+1}$ that are used in Theorem 2 are unchanged by a unitary similarity transformation on $A$, hence $(i)$ follows.

(ii) $\sigma\left(A^{T}\right)=\sigma(A)$ and $A^{T}$ will transform $W_{k}$ and $V_{k}$ into $W_{k}^{T}$ and $-V_{k}^{*}$, respectively, which leave Theorem 2 invariant.

(iii) $\sigma\left(A^{*}\right)=\overline{\sigma(A)}$ and $A^{*}$ will transform $V_{k}$ into $-V_{k}$ but $A^{*}-\lambda I_{n}$ gives a $W_{k}\left(A^{*} ; \lambda\right)=$ $\Delta_{k}-Y_{k}-\lambda I_{k}=\left(\Delta_{k}+Y_{k}-\bar{\lambda} I_{k}\right)^{*}=\left(W_{k}(A ; \bar{\lambda})\right)^{*}$ so Theorem 2 becomes a statement for $\bar{\lambda}$ if $\lambda \in \sigma\left(A^{*}\right)$.

(iv) $A+b I_{n}$ leaves $V_{k}$ unchanged and replaces $-\lambda I_{k}$ by $(b-\lambda) I_{k}$ in $W_{k}$. For $r>0, \Gamma_{k}(r A)=$ $\left\{(s+i t) / r \in \Gamma_{k}(A)\right\}=r \Gamma_{k}(A)$ because both sides of (6) in Theorem 2 then scale as $r^{2 k+1}$.

Next we give some illustrations of Proposition 3. In Figure 2 we depict the curve $\Gamma_{2}(A)$ defined by having equality in (19) for three random $5 \times 5$ complex matrices with elements that have real and imaginary parts between -1 and 1 , and compare it with the cubic curve $\Gamma_{1}(A)$ defined by having equality in (16) [1]. The eigenvalues are marked by small boxes. It is usually the case that the new curve is a strict improvement, i.e., all points in $\mathbb{C}$ satisfying the inequality for $k=2$ also satisfy the inequality for $k=1$. However, we shall see below that this is not always the case.
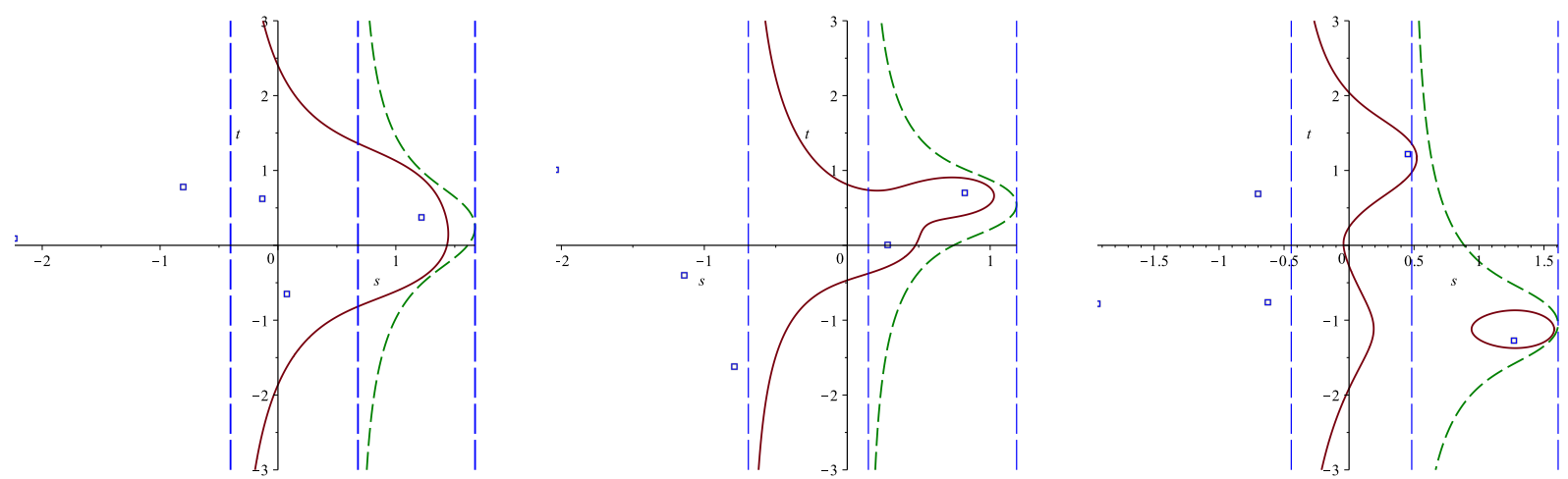

Figure 2: The curves $\Gamma_{2}(A)$ (solid) and $\Gamma_{1}(A)$ (dashed) for three complex $5 \times 5$ matrices. The vertical lines are $s=\delta_{3}, s=\delta_{2}$ and $s=\delta_{1}$.

In Figure 3 we have used six real random $5 \times 5$ matrices with elements between -1 and 1 to show some more possible configurations. From simulations it is clear that for random matrices 

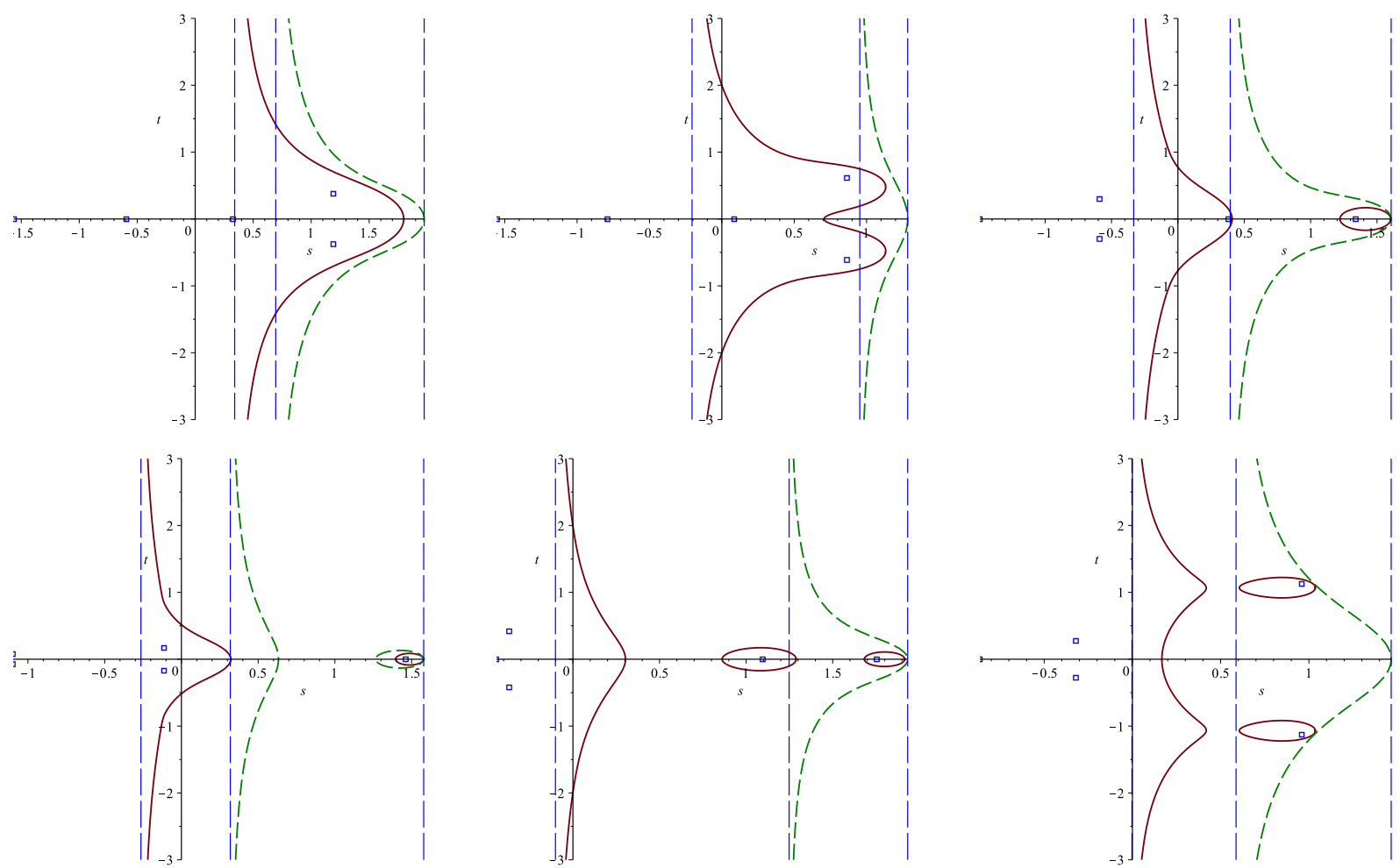

Figure 3: The curves $\Gamma_{2}(A)$ (solid) and $\Gamma_{1}(A)$ (dashed) for six real $5 \times 5$ matrices. The vertical lines are $s=\delta_{3}, s=\delta_{2}$ and $s=\delta_{1}$.

the plots of Figure 3 appear with decreasing probability. The first plots, with less interesting topology, appear more frequently but we shall see how one can construct matrices with all types of curves that are seen in the figure. In these figures the $k=2$ case is a strict improvement on the $k=1$ case.

It is clear that in these figures $s=\delta_{3}$ is an asymptote to the curve $\Gamma_{2}(A)$, and $s=\delta_{2}$ is an asymptote to $\Gamma_{1}(A)$, as stated in [1]. One can generalize this observation and prove it for arbitrary $k$.

Theorem 5. The curve $\Gamma_{k}(A):\left|\operatorname{det} W_{k}\right|^{2}\left(s-\delta_{k+1}\right)=\left(\sigma_{1}\left(V_{k}\right)\right)^{2} \lambda_{\max }\left(H\left(\operatorname{det} W_{k} \operatorname{adj}\left(W_{k}^{*}\right)\right)\right)$ has $s=\delta_{k+1}$ as an asymptote, and there are no points on the curve with $s<\delta_{k+1}$.

Proof. For $s<\delta_{k}, H\left(W_{k}\right)=\operatorname{diag}\left(\delta_{1}-s, \ldots, \delta_{k}-s\right)$ is positive definite which implies that $H\left(W_{k}^{-1}\right)$ is positive definite [4]. Then $\left|\operatorname{det} W_{k}\right|>0$ and $\lambda_{\max }\left(H\left(\operatorname{det} W_{k} \operatorname{adj}\left(W_{k}^{*}\right)\right)\right)>0$, so $s<\delta_{k+1}$ is not possible. Furthermore, if $V_{k} \neq 0$ we see that $\left|\operatorname{det} W_{k}\right| \rightarrow \infty$ is needed as $s \rightarrow \delta_{k+1}^{+}$and this implies $|t| \rightarrow \infty$. If $V_{k}=0$ then $s=\delta_{k+1}$ is the curve $\Gamma_{k}(A)$ except for isolated points given by $\operatorname{det} W_{k}=0$ (and $A$ is unitarily similar to a direct sum of $\Delta_{k}+Y_{k}$ and some $\left.B \in \mathcal{M}_{n-k}(\mathbb{C})\right)$.

Let $k=2$ again. For $s<\delta_{2}, H\left(W_{2}^{-1}\right)$ is positive definite which means that both eigenvalues of $M_{2}=H\left(\operatorname{det} W_{2} a d j\left(W_{2}^{*}\right)\right)$ are positive. By (21) and (22), $2 \lambda_{\min }\left(M_{2}\right)=m_{1}+m_{3}-$ $\sqrt{\left(m_{1}-m_{3}\right)^{2}+4\left|m_{2}\right|^{2}}=\operatorname{Tr} M_{2}-\sqrt{\left(\operatorname{Tr} M_{2}\right)^{2}-4 \operatorname{det} M_{2}}$. Denote by $\gamma_{2}(A)$ the curve

$$
\left|\operatorname{det} W_{2}\right|^{2}\left(s-\delta_{3}\right)=\left(\sigma_{1}\left(V_{2}\right)\right)^{2} \lambda_{\min }\left(M_{2}\right) \text {. }
$$


Then $\gamma_{2}(A)$ must be located to the left of $\Gamma_{2}(A)$ but still to the right of $s=\delta_{3}$, and with $s=\delta_{3}$ as an asymptote. With $K_{2}=\left(\sigma_{1}\left(V_{2}\right)\right)^{2}$ and $2 \lambda_{\max }\left(M_{2}\right)=\operatorname{Tr} M_{2}+\sqrt{\left(\operatorname{Tr} M_{2}\right)^{2}-4 \operatorname{det} M_{2}}$, the curves $\Gamma_{2}(A)$ and $\gamma_{2}(A)$ are given by $2\left|\operatorname{det} W_{2}\right|^{2}\left(s-\delta_{3}\right)-K_{2} \operatorname{Tr} M_{2}= \pm K_{2} \sqrt{\left(\operatorname{Tr} M_{2}\right)^{2}-4 \operatorname{det} M_{2}}$, with the plus sign for $\Gamma_{2}(A)$ and the minus sign for $\gamma_{2}(A)$. Squaring implies

$$
4\left|\operatorname{det} W_{2}\right|^{4}\left(s-\delta_{3}\right)^{2}-4 K_{2} \operatorname{Tr} M_{2}\left|\operatorname{det} W_{2}\right|^{2}\left(s-\delta_{3}\right)=-4 K_{2}^{2} \operatorname{det} M_{2},
$$

which is a polynomial curve $\Gamma_{2}(A) \cup \gamma_{2}(A)$ for $s$ and $t$. The components $\Gamma_{2}(A)$ and $\gamma_{2}(A)$ of 26. connect at points where $\left(\operatorname{Tr} M_{2}\right)^{2}=4 \operatorname{det} M_{2}$ and at infinity.

We illustrate the curve in $(26)$ in Figure 4 for two random complex $5 \times 5$ matrices, for which the two components $\Gamma_{2}(A)$ and $\gamma_{2}(A)$ are disjoint, and for the matrix

$$
\hat{A}=\left(\begin{array}{cccc}
2 & 0 & 0 & -1.01 \\
0 & 1 & 0 & 0 \\
0 & 0 & 0 & -1 \\
1.01 & 0 & 1 & 0
\end{array}\right) .
$$

We see that $s=\delta_{3}$ is an asymptote for the two components. For $\hat{A}$ we see that the two components meet at some points and that they are non-smooth there. We shall comment more on this in the next section.
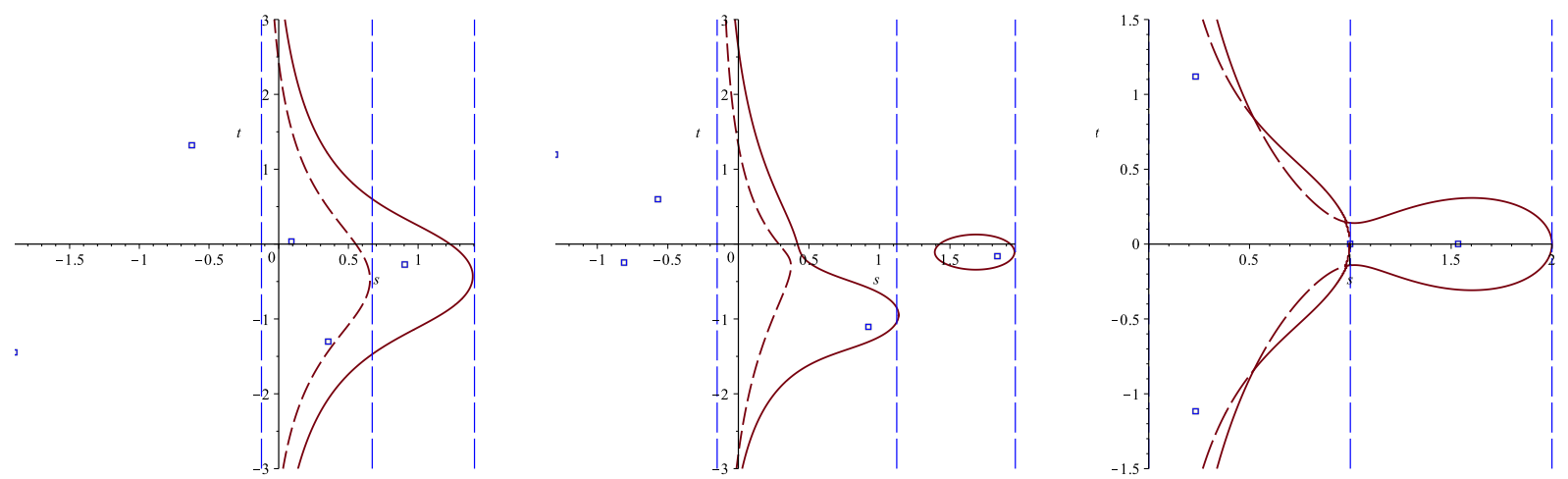

Figure 4: The two parts $\Gamma_{2}(A)$ (solid) and $\gamma_{2}(A)$ (dashed) of the curve given by (26), for two random complex $5 \times 5$ matrices (left and center), and for the matrix $\hat{A}$ in 27 (right). The vertical lines are $s=\delta_{3}, s=\delta_{2}$ and $s=\delta_{1}$.

Although in Figures 2 and 3 the curves $\Gamma_{2}(A)$ give a strict improvement compared with the cubic curves $\Gamma_{1}(A)$, it is not uncommon that for some region in the band $\delta_{2}<s<\delta_{1}$, the cubic curve is better.

In Figure 5 we show three cases, the first two subfigures refer to a complex and a real $5 \times 5$ matrix, respectively, and the third to the real matrix $\tilde{A}$ in 29 , where in some region the cubic curve $\Gamma_{1}(A)$ gives more restrictions on the spectrum than $\Gamma_{2}(A)$. We see that we can even have a closed loop on $\Gamma_{1}(A)$ without having a closed loop on $\Gamma_{2}(A)$.

A general analysis of this situation is complex but we can state some sufficient conditions for the cubic curve $\Gamma_{1}(A)$ to be more restrictive. Assume for simplicity that $A \in \mathcal{M}_{n}(\mathbb{R})$. Then the situation will occur if for some value of $s$ the value of $|t|$ is smaller, or maybe non-existent, on $\Gamma_{1}(A)$ than on $\Gamma_{2}(A)$, since $s=\delta_{2}$ is an asymptote for $\Gamma_{1}(A)$. 

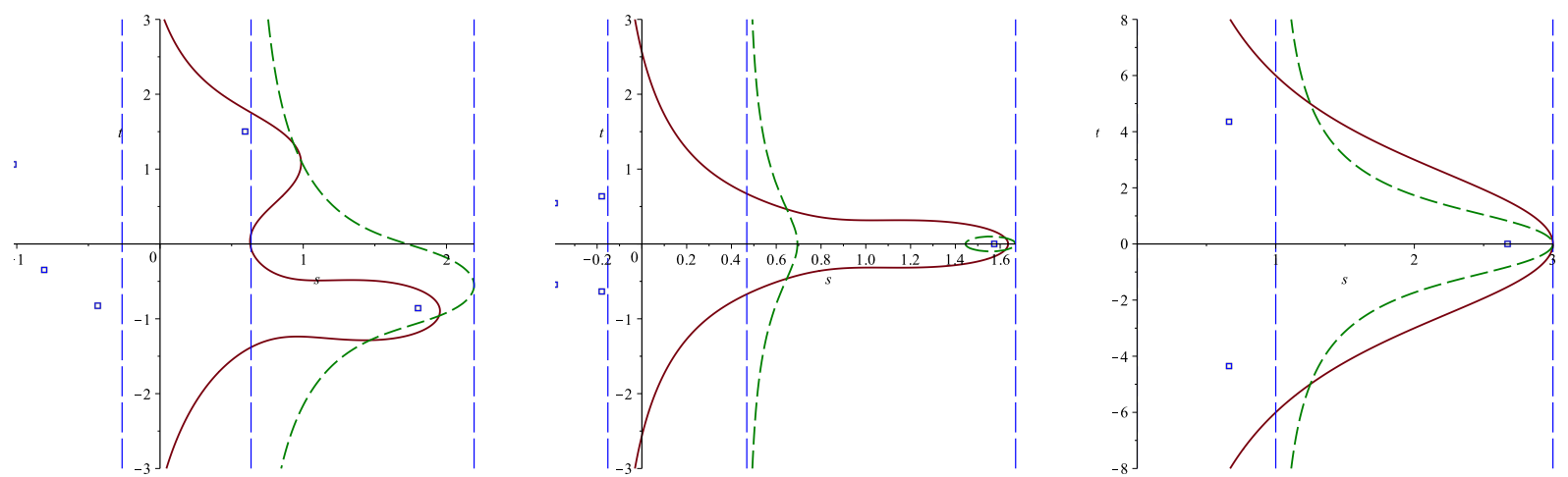

Figure 5: The curves $\Gamma_{2}(A)$ (solid) and $\Gamma_{1}(A)$ (dashed) for a complex $5 \times 5$ matrix (left), a real $5 \times 5$ matrix (center), and the matrix $\tilde{A}$ in 29 (right). The vertical lines are $s=\delta_{3}, s=\delta_{2}$ and $s=\delta_{1}$.

Assuming $A \in \mathcal{M}_{n}(\mathbb{R})$, we have $\alpha=0$ and $K_{1}=\left\|S(A) \mathbf{u}_{1}\right\|_{2}^{2}$, so by 16 the cubic curve $\Gamma_{1}(A)$ becomes

$$
\left[\left(s-\delta_{1}\right)^{2}+t^{2}\right]\left(s-\delta_{2}\right)=K_{1}\left(\delta_{1}-s\right),
$$

which gives $t^{2}=K_{1}\left(\delta_{1}-s\right) /\left(s-\delta_{2}\right)-\left(s-\delta_{1}\right)^{2}$. For $k=2$ the corresponding analysis is harder, we therefore choose to compare the values of $t^{2}$ on $\Gamma_{1}(A)$ and $\Gamma_{2}(A)$, denoted by $t_{1}^{2}$ and $t_{2}^{2}$, respectively, for the value $\tilde{s}=\left(\delta_{1}+\delta_{2}\right) / 2$ of $s$, and check when $t_{1}^{2}<t_{2}^{2}$. For $k=1$, on $\Gamma_{1}(A)$, we get $t_{1}^{2}=K_{1}-\left(\delta_{1}-\delta_{2}\right)^{2} / 4$. For $k=2$, on $\Gamma_{2}(A)$, with $\alpha=0, \beta=0, \gamma \in \mathbb{R}$ and $s=\tilde{s}$, Proposition 3 gives

$$
\left[\left(\frac{\delta_{1}-\delta_{2}}{2}\right)^{2}+t_{2}^{2}-\gamma^{2}\right]^{2}\left(\frac{\delta_{1}+\delta_{2}}{2}-\delta_{3}\right)=\frac{K_{2}}{2}\left|\left(\delta_{1}-\delta_{2}\right)\left[\left(\frac{\delta_{1}-\delta_{2}}{2}\right)^{2}+t_{2}^{2}-\gamma^{2}\right]\right|,
$$

where

$2 K_{2}=\left\|S(A) \mathbf{u}_{1}\right\|_{2}^{2}+\left\|S(A) \mathbf{u}_{2}\right\|_{2}^{2}-2 \gamma^{2}+\sqrt{\left(\left\|S(A) \mathbf{u}_{1}\right\|_{2}^{2}-\left\|S(A) \mathbf{u}_{2}\right\|_{2}^{2}\right)^{2}+4\left(\left(S(A) \mathbf{u}_{2}\right)^{*} S(A) \mathbf{u}_{1}\right)^{2}}$.

This implies that $t_{2}^{2}=\gamma^{2}-\left(\frac{\delta_{1}-\delta_{2}}{2}\right)^{2}$ or $t_{2}^{2}=\gamma^{2}-\left(\frac{\delta_{1}-\delta_{2}}{2}\right)^{2} \pm K_{2}\left(\delta_{1}-\delta_{2}\right) /\left(\delta_{1}+\delta_{2}-2 \delta_{3}\right)$. Since by 18, $K_{1}=\gamma^{2}+\left\|\mathbf{v}_{1}\right\|_{2}^{2} \geq \gamma^{2}$, we have $t_{1}^{2} \geq \gamma^{2}-\left(\frac{\delta_{1}-\delta_{2}}{2}\right)^{2} \geq \gamma^{2}-\left(\frac{\delta_{1}-\delta_{2}}{2}\right)^{2}-K_{2}\left(\delta_{1}-\delta_{2}\right) /\left(\delta_{1}+\delta_{2}-2 \delta_{3}\right)$. The question is therefore when $t_{1}^{2}<\gamma^{2}-\left(\frac{\delta_{1}-\delta_{2}}{2}\right)^{2}+K_{2}\left(\delta_{1}-\delta_{2}\right) /\left(\delta_{1}+\delta_{2}-2 \delta_{3}\right)$ is possible. Since $t_{1}^{2}=K_{1}-\left(\frac{\delta_{1}-\delta_{2}}{2}\right)^{2}$, we need to have $K_{1}<\gamma^{2}+K_{2}\left(\delta_{1}-\delta_{2}\right) /\left(\delta_{1}+\delta_{2}-2 \delta_{3}\right)$. Expressed in terms of the vectors $\mathbf{v}_{1}$ and $\mathbf{v}_{2}$ of (18), and using (23), this inequality is

$$
\left\|\mathbf{v}_{1}\right\|_{2}^{2}<K_{2} \cdot \frac{\delta_{1}-\delta_{2}}{\delta_{1}+\delta_{2}-2 \delta_{3}} ; 2 K_{2}=\left\|\mathbf{v}_{1}\right\|_{2}^{2}+\left\|\mathbf{v}_{2}\right\|_{2}^{2}+\sqrt{\left(\left\|\mathbf{v}_{1}\right\|_{2}^{2}-\left\|\mathbf{v}_{2}\right\|_{2}^{2}\right)^{2}+4\left(\mathbf{v}_{2}^{*} \mathbf{v}_{1}\right)^{2}}
$$

If the vectors $\mathbf{v}_{1}$ and $\mathbf{v}_{2}$ are orthogonal, $K_{2}=\max \left\{\left\|\mathbf{v}_{1}\right\|_{2}^{2},\left\|\mathbf{v}_{2}\right\|_{2}^{2}\right\}$, if they are parallel, $K_{2}=$ $\left\|\mathbf{v}_{1}\right\|_{2}^{2}+\left\|\mathbf{v}_{2}\right\|_{2}^{2}$. Also, $0<\left(\delta_{1}-\delta_{2}\right) /\left(\delta_{1}+\delta_{2}-2 \delta_{3}\right)=\left(\delta_{1}-\delta_{2}\right) /\left(\delta_{1}-\delta_{2}+2\left(\delta_{2}-\delta_{3}\right)\right)<1$ and closer to 1 if $\delta_{2}-\delta_{3}$ is small compared to $\delta_{1}-\delta_{2}$. Thus matrices with $\left\|\mathbf{v}_{1}\right\|_{2}$ small compared to $\left\|\mathbf{v}_{2}\right\|_{2}$ and $\delta_{2}-\delta_{3}$ small compared to $\delta_{1}-\delta_{2}$ will have the desired property.

The $3 \times 3$ matrix

$$
\tilde{A}=\left(\begin{array}{ccc}
3 & 0 & -2 \\
0 & 1 & -4 \\
2 & 4 & 0
\end{array}\right)
$$


has $\delta_{1}=3, \delta_{2}=1, \delta_{3}=0, \mathbf{v}_{1}, \mathbf{v}_{2} \in \mathbb{R},\left\|\mathbf{v}_{1}\right\|_{2}=2,\left\|\mathbf{v}_{2}\right\|_{2}=4, \mathbf{v}_{2}^{*} \mathbf{v}_{1}=8, K_{1}=4$, and $K_{2}=20$. We get $\tilde{s}=2$ and $t_{1}^{2}=3<9=t_{2}^{2}$, and we see the curves in the last subfigure of Figure 5

In these cases, one may of course combine $\Gamma_{1}(A)$ and $\Gamma_{2}(A)$ and use the intersection of the two regions to minimize the region for the spectrum. We also emphasize that the condition 28 is sufficient but not necessary for the two curves to cross since we assumed $A$ real and $s=\tilde{s}=\left(\delta_{1}+\delta_{2}\right) / 2$.

\section{Topologically interesting examples}

To analyze the curves $\Gamma_{k}(A)$ for larger values of $k$ is in general hard, the complicated dependence of $\lambda_{\max }\left(H\left(\operatorname{det} W_{k} \operatorname{adj}\left(W_{k}^{*}\right)\right)\right)$ on $s$ and $t$ being a main cause. Each element of $\operatorname{adj}\left(W_{k}^{*}\right)$ is a $(k-1) \times(k-1)$ minor of $W_{k}^{*}$. If we assume that we have a matrix $A$ such that $W_{k}$ is diagonal, then the curves are easier to analyze and we can already in this case see interesting types of behavior of $\Gamma_{k}(A)$ that can occur. Using the notation of (3) and (4), we therefore assume that $A$ has the form

$$
A=\left(\begin{array}{cc}
\Delta_{k} & -V_{k}^{*} \\
V_{k} & \tilde{A}_{k}
\end{array}\right)
$$

where $\Delta_{k}=\operatorname{diag}\left(\delta_{1}, \ldots, \delta_{k}\right) \in \mathcal{M}_{k}(\mathbb{R})$ and $\tilde{A}_{k}=\tilde{\Delta}_{k}+\tilde{Y}_{k}$. This means that in (3) and (4) we have assumed $Y_{k}=0$ and $U=I_{n}$. Then, by (5), $W_{k}=\Delta_{k}-\lambda I_{k}$ is diagonal with

$$
\operatorname{det} W_{k}=\prod_{r=1}^{k}\left(\delta_{r}-\lambda\right)
$$

We get

$$
\begin{aligned}
\operatorname{det} W_{k} \operatorname{adj}\left(W_{k}^{*}\right) & =\operatorname{det} W_{k} \operatorname{adj}\left[\operatorname{diag}\left(\delta_{1}-\bar{\lambda}, \ldots, \delta_{k}-\bar{\lambda}\right)\right] \\
& =\operatorname{det} W_{k} \operatorname{diag}\left(\prod_{r=2}^{k}\left(\delta_{r}-\bar{\lambda}\right), \ldots, \prod_{r=1}^{k-1}\left(\delta_{r}-\bar{\lambda}\right)\right) \\
& =\operatorname{diag}\left(\left(\delta_{1}-\lambda\right) \prod_{r=2}^{k}\left|\delta_{r}-\lambda\right|^{2}, \ldots,\left(\delta_{k}-\lambda\right) \prod_{r=1}^{k-1}\left|\delta_{r}-\lambda\right|^{2}\right) .
\end{aligned}
$$

With $\lambda=s+i t$, the Hermitian part is

$$
M_{k}=H\left(\operatorname{det} W_{k} \operatorname{adj}\left(W_{k}^{*}\right)\right)=\operatorname{diag}\left(\left(\delta_{1}-s\right) \prod_{r=2}^{k}\left|\delta_{r}-\lambda\right|^{2}, \ldots,\left(\delta_{k}-s\right) \prod_{r=1}^{k-1}\left|\delta_{r}-\lambda\right|^{2}\right) .
$$

The eigenvalues of $M_{k}$ are its diagonal elements and we need to determine the largest eigenvalue. Suppose that $j<i$, so $\delta_{j} \geq \delta_{i}$, and compare the corresponding diagonal elements $\left(M_{k}\right)_{j j}$ and $\left(M_{k}\right)_{i i}$ of $M_{k}$. We have $\left(\delta_{j}-s\right) \prod_{r \neq j}\left|\delta_{r}-\lambda\right|^{2} \geq\left(\delta_{i}-s\right) \prod_{r \neq i}\left|\delta_{r}-\lambda\right|^{2}$ if $\left(\delta_{j}-s\right)\left|\delta_{i}-\lambda\right|^{2} \geq$ $\left(\delta_{i}-s\right)\left|\delta_{j}-\lambda\right|^{2}$. This means $\left(\delta_{j}-s\right)\left(\left(\delta_{i}-s\right)^{2}+t^{2}\right) \geq\left(\delta_{i}-s\right)\left(\left(\delta_{j}-s\right)^{2}+t^{2}\right)$, or, equivalently $\left(\delta_{j}-s\right)\left(\delta_{i}-s\right) \leq t^{2}$. Writing it as

$$
\left(s-\frac{\delta_{j}+\delta_{i}}{2}\right)^{2}-t^{2} \leq\left(\frac{\delta_{j}-\delta_{i}}{2}\right)^{2},
$$


we see that equality is attained at the hyperbola with centerpoint at $\left(\frac{\delta_{j}+\delta_{i}}{2}\right)^{2}$ on the real axis, passing through the real axis at $\delta_{j}$ and $\delta_{i}$, and with asymptotes of slope \pm 1 .

Suppose we are in the region $\delta_{k+1} \leq s \leq \delta_{1}$. No components bending in the same direction (left or right) of all the hyperbolas for all pairs $j, i$ will cross each other since they have the same asymptotic slope. Of all hyperbolas formed from $\delta_{1}$ and $\delta_{j}, j=2, \ldots, k$, it is the left component of the one formed from $\delta_{1}$ and $\delta_{2}$ which gives the left boundary of the region where the first diagonal element $\left(M_{k}\right)_{11}$ of $M_{k}$ equals $\lambda_{\max }\left(M_{k}\right)$. Next, to the left of this curve, the second diagonal element $\left(M_{k}\right)_{22}$ will be $\lambda_{\max }\left(M_{k}\right)$ until we reach the left component of the hyperbola formed from $\delta_{2}$ and $\delta_{3}$ and so on. We illustrate the situation in Figure6. In the first subfigure we show all six hyperbolas in a case with $k=3, \delta_{1}=5, \delta_{2}=3.5, \delta_{3}=1$ and $\delta_{4}=0$. The hyperbolas related to $\left(\delta_{1}, \delta_{2}\right),\left(\delta_{1}, \delta_{3}\right)$ and $\left(\delta_{1}, \delta_{4}\right)$ are solid, the ones of $\left(\delta_{2}, \delta_{3}\right)$ and $\left(\delta_{2}, \delta_{4}\right)$ dashed, and the one of $\left(\delta_{3}, \delta_{4}\right)$ dotted. In the second subfigure, we have $k=4, \delta_{1}=5, \delta_{2}=3.5, \delta_{3}=3, \delta_{4}=1$ and $\delta_{5}=0$, and we show those components of all 10 hyperbolas that separate four regions of different diagonal elements of $M_{4}$ being $\lambda_{\max }\left(M_{4}\right)$ for $\delta_{5} \leq s \leq \delta_{1}$.
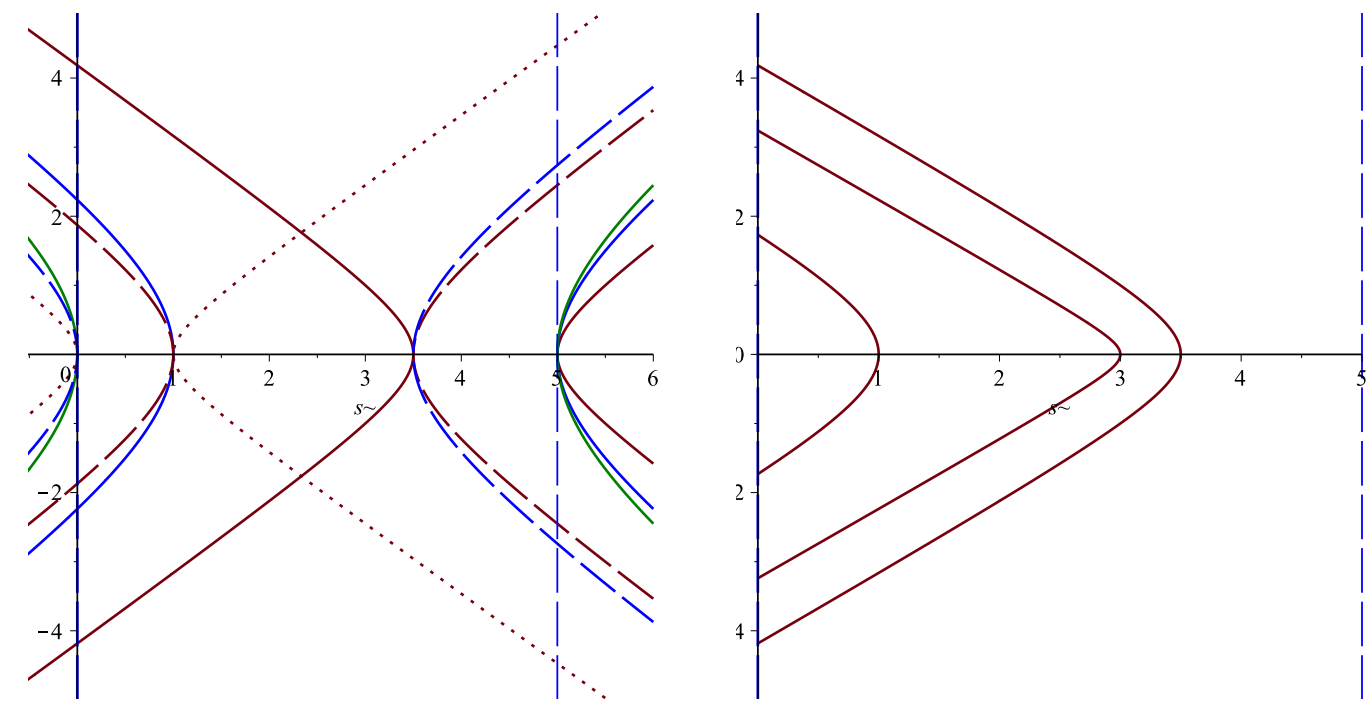

Figure 6: All six hyperbolas for a $k=3$ case (left) and the three region separating hyperbola components for a $k=4$ case (right).

With $\sigma_{1}\left(V_{k}\right)=\varepsilon$, the curve $\Gamma_{k}(A)$ of Theorem 2 in each region becomes

$$
\prod_{r=1}^{k}\left|\delta_{r}-\lambda\right|^{2}\left(s-\delta_{k+1}\right)=\varepsilon^{2}\left(\delta_{j}-s\right) \prod_{r=1, r \neq j}^{k}\left|\delta_{r}-\lambda\right|^{2},
$$

which is $\left|\delta_{j}-\lambda\right|^{2}\left(s-\delta_{k+1}\right)=\varepsilon^{2}\left(\delta_{j}-s\right)$ or

$$
\left[\left(\delta_{j}-s\right)^{2}+t^{2}\right]\left(s-\delta_{k+1}\right)=\varepsilon^{2}\left(\delta_{j}-s\right),
$$

which is a curve of degree 3 . Thus, our entire curve $\Gamma_{k}(A)$ consists of different cubic curves which are connected in a continuous but maybe non-smooth way at the hyperbolas that separates the regions. Note that no details of $V_{k}$ or $\tilde{A}_{k}$ in 30 are needed to construct the curve.

Putting $t=0$ in (31), we observe that $s=\delta_{j}$ or

$$
s=s_{ \pm}=\frac{\delta_{j}+\delta_{k+1}}{2} \pm \sqrt{\left(\frac{\delta_{j}-\delta_{k+1}}{2}\right)^{2}-\varepsilon^{2}}
$$


For $1 \leq j \leq k-1$, the value $s_{+}$is between $\delta_{j+1}$ and $\delta_{j}$ if $s_{+} \geq \delta_{j+1}$, which happens if $\varepsilon \leq \varepsilon_{j}$, where

$$
\varepsilon_{j}=\sqrt{\left(\delta_{j+1}-\delta_{k+1}\right)\left(\delta_{j}-\delta_{j+1}\right)} .
$$

Note that $\varepsilon_{j} \leq\left(\left(\delta_{j+1}-\delta_{k+1}\right)+\left(\delta_{j}-\delta_{j+1}\right)\right) / 2=\left(\delta_{j}-\delta_{k+1}\right) / 2$ so $s_{ \pm}$exist (real) for $\varepsilon \leq \varepsilon_{j}$. Then there will be a closed loop passing through $s=s_{+}$and $s=\delta_{j}$. If also $s_{-} \geq \delta_{j+1}$ (which could only happen if $\left.\delta_{j+1} \leq\left(\delta_{j}+\delta_{k+1}\right) / 2\right)$, then $s_{-}$will be on the unbounded component of $\Gamma_{k}(A)$ (and any loops to the left have already merged with the unbounded component).

In the region most to the left, where $s \leq \delta_{k}$, for $\varepsilon<\varepsilon_{k}=\left(\delta_{k}-\delta_{k+1}\right) / 2$ we have $s_{ \pm}>\delta_{k+1}$ and there is a loop through $\delta_{k}$ and $s_{+}$while the unbounded component of $\Gamma_{k}(A)$ passes through $s=s_{-}$. For $\varepsilon=\varepsilon_{k}$ the loop connects to the unbounded component. For $\varepsilon>\varepsilon_{k}$ there is only one component, it is unbounded and passes through $s=\delta_{k}$.

In general, because of the different hyperbolas, the unbounded component of $\Gamma_{k}(A)$ passes through several regions as $|t|$ grows and $s \rightarrow \delta_{k+1}^{+}$, so it may have points where it is not smooth.

An example of this is provided by the matrix $\hat{A}$ in 27 . In the last subfigure of Figure 4 we see the non-smoothness of $\Gamma_{2}(\hat{A})$ which occurs at points of the hyperbola $(s-3 / 2)^{2}+t^{2}=1 / 4$. We have $\varepsilon=1.01$ and the four crossings where $\lambda_{\min }\left(M_{2}\right)=\lambda_{\max }\left(M_{2}\right)$, so $\Gamma_{2}(\hat{A})$ and $\gamma_{2}(\hat{A})$ of 25 meet, are readily calculated to have coordinates $s=s_{1,2}=\left(3 \pm \sqrt{9-8 \varepsilon^{2}}\right) / 4 \approx 0.75 \pm 0.229$, each with two corresponding $t$-values $t= \pm \sqrt{s_{1,2}^{2}-3 s_{1,2}+2}$. For $s \in[0,0.521] \cup[0.979,2]$ the diagonal element $\left(M_{2}\right)_{11}=(2-s)\left[(1-s)^{2}+t^{2}\right]$ of $M_{2}$ is its largest eigenvalue, and for $s \in[0.521,0.979],\left(M_{2}\right)_{22}=(1-s)\left[(2-s)^{2}+t^{2}\right]$ is the largest, and these non-smooth changes take place at the crossing with the hyperbola.

With the above relations between $\delta_{1}, \ldots, \delta_{k+1}$ and $\varepsilon_{1}, \ldots, \varepsilon_{k}$, one can construct matrices that give curves $\Gamma_{k}(A)$ with desired topologies.

As a first example, let $k=2$ and define two matrices

$$
A=\left(\begin{array}{cccc}
1.36 & 0 & 0 & -\varepsilon / 2 \\
0 & 1 & -\varepsilon & 0 \\
0 & \varepsilon & 0 & -0.25 \\
\varepsilon / 2 & 0 & 0.25 & 0
\end{array}\right), B=\left(\begin{array}{cccc}
1.16 & 0 & 0 & -\varepsilon / 2 \\
0 & 1 & -\varepsilon & 0 \\
0 & \varepsilon & 0 & -0.25 \\
\varepsilon / 2 & 0 & 0.25 & 0
\end{array}\right)
$$

Both have $\delta_{3}=0, \delta_{2}=1$, and $\sigma_{1}\left(V_{2}\right)=\varepsilon$. Then $\varepsilon_{2}=\left(\delta_{2}-\delta_{3}\right) / 2=0.5$, and, by (32), $\varepsilon_{1}=\sqrt{\delta_{1}-1}$. For $A, \varepsilon_{1}=0.6$, and for $B, \varepsilon_{1}=0.4$. For small $\varepsilon$, both matrices will have curves $\Gamma_{2}(A), \Gamma_{2}(B)$ with two closed loops enclosing one eigenvalue each. For $A$, when $\varepsilon$ increases, first at $\varepsilon=0.5$ the left loop merges with the unbounded component, and then at $\varepsilon=0.6$ the remaining loop merges with the unbounded component. For $B$, when $\varepsilon$ increases, first at $\varepsilon=0.4$ the two loops merge into one that encloses two eigenvalues, and at $\varepsilon=0.5$ this loop merges with the unbounded component. In Figure 7 we show first $\Gamma_{2}(A)$ for $\mathrm{A}$ for $\varepsilon=0.45,0.55,0.65$ and below $\Gamma_{2}(B)$ for $B$ for $\varepsilon=0.35,0.45,0.55$. The eigenvalues are marked by small boxes. In the last plot of $A$ and in the last two plots of $B$, one sees clearly the non-smooth points on the curves which are located at the crossings with the left-bending component of the hyperbola that passes through $s=1, t=0$.

For larger values of $k$, one can also choose values of $\delta_{1}, \ldots, \delta_{k+1}$ such that the mergers of neighboring loops of $\Gamma_{k}(A)$ come in any desired order. If we want all mergers to occur for the same $\varepsilon$, i.e., if $\varepsilon_{1}=\varepsilon_{2}=\cdots=\varepsilon_{k}=\left(\delta_{k}-\delta_{k+1}\right) / 2$, we can also obtain this. By (32) this will happen if, given $\delta_{k+1}$ and $\delta_{k}$, we choose iteratively for decreasing $j=k, \ldots, 2$, 

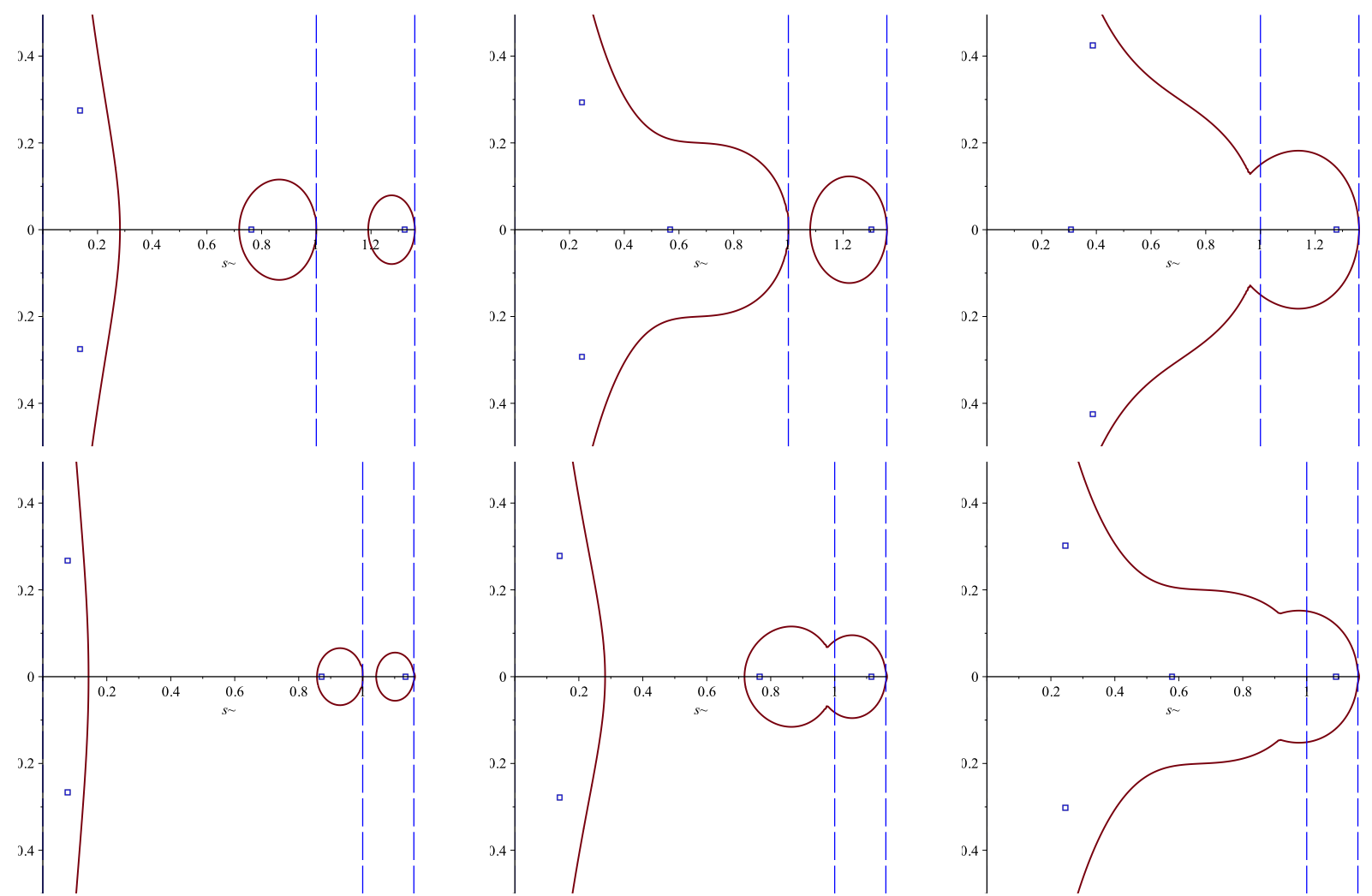

Figure 7: The curves $\Gamma_{2}(A)$ (top) and $\Gamma_{2}(B)$ (bottom) for the matrices $A$ and $B$ in $(33)$ for increasing values of $\varepsilon$.

$\delta_{j-1}=\delta_{j}+\left(\delta_{k}-\delta_{k+1}\right)^{2} /\left(4\left(\delta_{j}-\delta_{k+1}\right)\right)$. As an example, we take $k=4, \delta_{5}=0$ and $\delta_{4}=1$. Then we get $\delta_{3}=5 / 4, \delta_{2}=29 / 20$ and $\delta_{1}=941 / 580$, and the simultaneous merger is at $\varepsilon=1 / 2$. Define a matrix

$$
C=\left(\begin{array}{cccccc}
941 / 580 & 0 & 0 & 0 & 0 & -\varepsilon / \sqrt{2} \\
0 & 29 / 20 & 0 & 0 & -\varepsilon / \sqrt{2} & 0 \\
0 & 0 & 5 / 4 & 0 & 0 & -\varepsilon / \sqrt{2} \\
0 & 0 & 0 & 1 & -\varepsilon / \sqrt{2} & 0 \\
0 & \varepsilon / \sqrt{2} & 0 & \varepsilon / \sqrt{2} & 0 & -0.25 \\
\varepsilon / \sqrt{2} & 0 & \varepsilon / \sqrt{2} & 0 & 0.25 & 0
\end{array}\right),
$$

which, since $V_{4} \in \mathcal{M}_{2,4}(\mathbb{R})$ has $\sigma_{1}\left(V_{4}\right)=\varepsilon$, has the desired properties. In Figure 8 we depict the curve $\Gamma_{4}(C)$ of $C$ for $\varepsilon=0.45,0.5,0.55$. In the last subfigure we see the non-smooth points on the curve located at the crossings with the hyperbolas.

Finally we demonstrate an interesting possibility. Let $k=2$ and

$$
F\left(\varepsilon_{1}, \varepsilon_{2}\right)=\left(\begin{array}{cccc}
5 & -\varepsilon_{2} & 0 & 0 \\
\varepsilon_{2} & 5 & -\varepsilon_{1} & 0 \\
0 & \varepsilon_{1} & 0 & -1 \\
0 & 0 & 1 & 0
\end{array}\right) .
$$



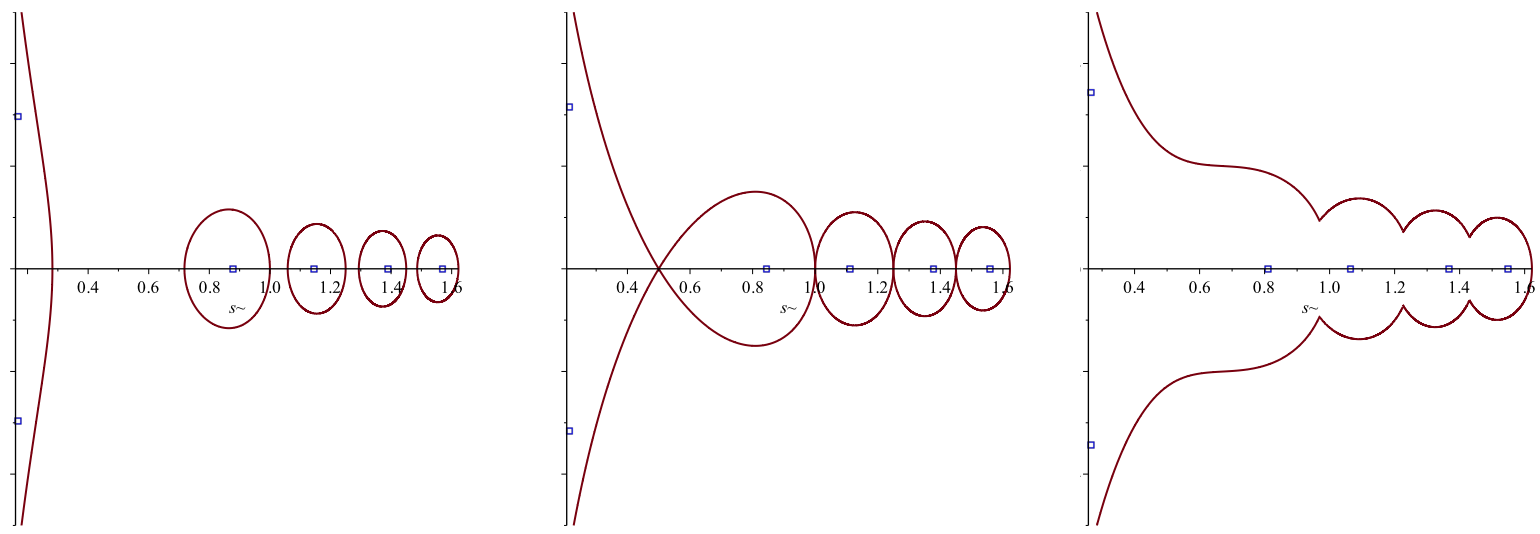

Figure 8: The curves $\Gamma_{4}(C)$ for the matrix $C$ in $(34)$ for increasing values of $\varepsilon$.

We start with $F(2.48,1.0)$ which has a pair of complex conjugated eigenvalues, each inside a loop of $\Gamma_{2}(F(2.48,1.0))$. If we decrease $\varepsilon_{2}$, the two loops will merge and form a new loop enclosing two eigenvalues. If instead we increase $\varepsilon_{1}$, each loop will connect to the unbounded component first. One can balance the parameters and obtain a situation where the loops approach each other and the unbounded component in such a way that an inner loop is formed that encloses a domain where no eigenvalue can be located. This is illustrated in Figure 9 where the matrices used are $F(2.48,1.0), F(2.48,0.66), F(2.52,1.0)$ and $F(2.52,0.66)$. On the top row the curves $\Gamma_{2}\left(F\left(\varepsilon_{1}, \varepsilon_{2}\right)\right)$ are illustrated, and on the bottom row the curves $\gamma_{2}\left(F\left(\varepsilon_{1}, \varepsilon_{2}\right)\right)$ of $(25)$, obtained by using $\lambda_{\min }\left(M_{2}\right)$, are added (dashed). The two curves together form a smooth algebraic curve given by (26), but break up at non-smooth points for each component. We observe that the curve $\gamma_{2}(A)$ in 25) may be non-connected and have a closed loop, compare with Figure 4.
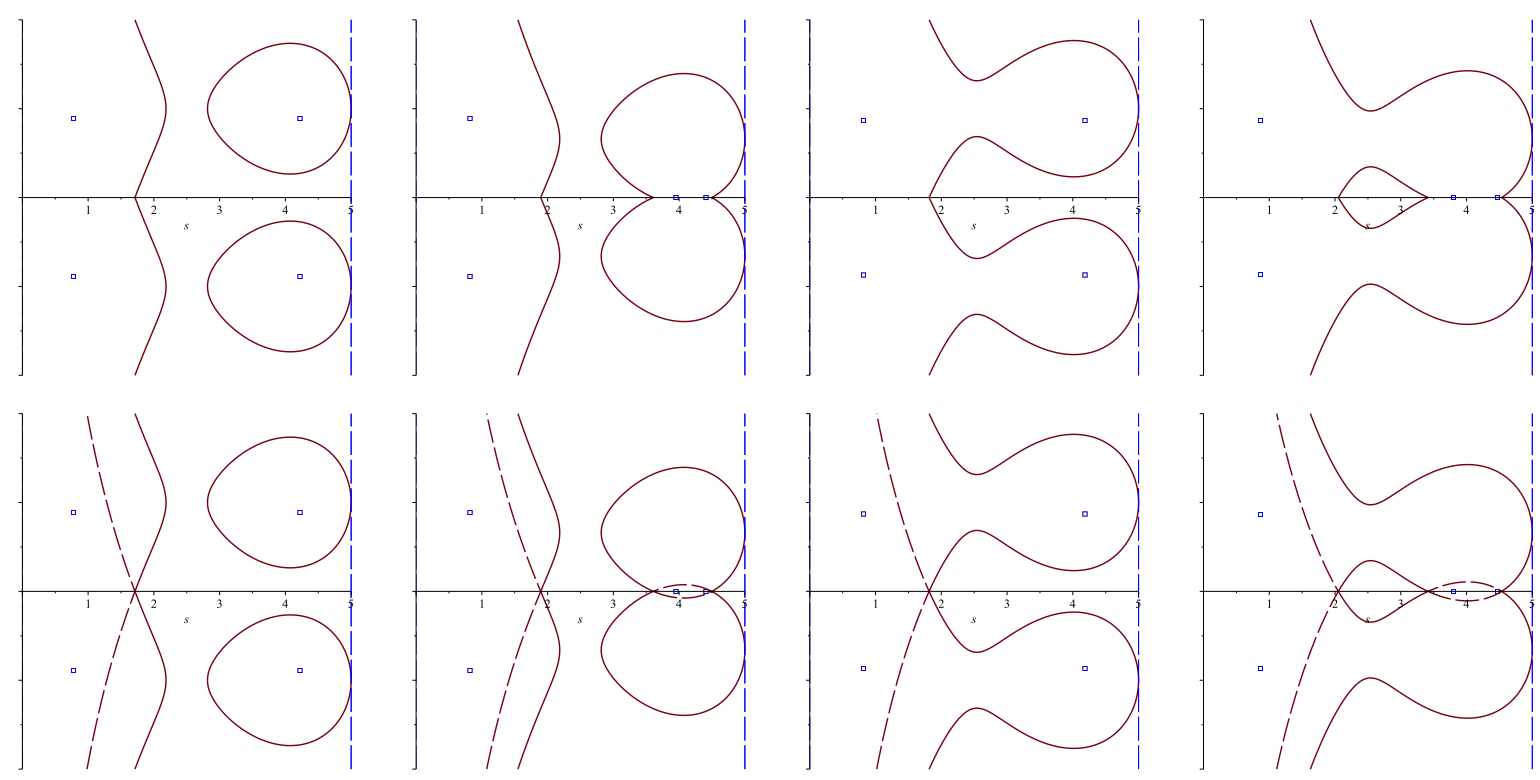

Figure 9: The curves $\Gamma_{2}\left(F\left(\varepsilon_{1}, \varepsilon_{2}\right)\right)$ for the matrices $F(2.48,1.0), F(2.48,0.66), F(2.52,1.0)$ and $F(2.52,0.66)$ in $(35)$ (first row). For $F(2.52,0.66)$, no eigenvalue can be inside the closed loop. On the second row of plots, the curves $\gamma_{2}\left(F\left(\varepsilon_{1}, \varepsilon_{2}\right)\right)$ are added (dashed). 


\section{Envelopes for the spectrum}

In the same way as for the lines used to bound the numerical range $F(A)[2$, 3] or the cubic curves $\Gamma_{1}(A)$ by Adam, Psarrakos and Tsatsomeros [1, 5, 6], we can apply our theorem to $e^{i \theta} A$ and obtain a curve that bounds $\sigma\left(e^{i \theta} A\right)$. Rotating that curve by $e^{-i \theta}$, we get a curve that bounds $\sigma(A)$. Doing this for all $\theta \in[0,2 \pi$ [ we get an infinite intersection of regions that contains $\sigma(A)$, and whose boundary is the envelope of such curves.

For a given matrix $A$, let $E_{k}\left(e^{i \theta} A\right)$ be all points $\lambda \in \mathbb{C}$ that satisfy

$$
\left|\operatorname{det} W_{k}\right|^{2}\left(\operatorname{Re}(\lambda)-\delta_{k+1}\right) \leq\left(\sigma_{1}\left(V_{k}\right)\right)^{2} \lambda_{\max }\left(H\left(\operatorname{det} W_{k} \operatorname{adj}\left(W_{k}^{*}\right)\right)\right),
$$

where $W_{k}=W_{k}(\theta), V_{k}=V_{k}(\theta)$ and $\delta_{k+1}=\delta_{k+1}(\theta)$ are constructed from $e^{i \theta} A$ as in Section 2 .

We denote the region that is the intersection over all $\theta \in\left[0,2 \pi\left[\right.\right.$ by $\mathcal{E}_{k}(A)$ :

Definition 2. $\mathcal{E}_{k}(A)=\bigcap_{\theta \in[0,2 \pi[} e^{-i \theta} E_{k}\left(e^{i \theta} A\right)$

The region $\mathcal{E}_{1}(A)$ was studied in detail in [5, 6] and we generalize some of their results. Recall that the $\ell$-rank numerical range of $A$ is $\Lambda_{\ell}(A)=\left\{\mu \in \mathbb{C} ; X^{*} A X=\mu I_{\ell}, X \in \mathcal{M}_{n, \ell}(\mathbb{C}), X^{*} X=\right.$ $\left.I_{\ell}\right\}=\bigcap_{\theta \in[0,2 \pi[} e^{-i \theta}\left\{s+i t ; s \leq \delta_{\ell}\left(e^{i \theta} A\right)\right\}$, and that in general $\sigma(A) \not \subset \Lambda_{\ell}(A)$ if $\ell \geq 2[6]$.

Proposition 6. Let $A \in \mathcal{M}_{n}(\mathbb{C})$. For the regions $\mathcal{E}_{k}(A)$ of the complex plane the following hold:

(i) $\sigma(A) \subset \mathcal{E}_{k}(A)$

(ii) $\mathcal{E}_{k}\left(\tilde{U}^{*} A \tilde{U}\right)=\mathcal{E}_{k}(A)$ if $\tilde{U}$ is unitary

(iii) $\mathcal{E}_{k}\left(A^{T}\right)=\mathcal{E}_{k}(A)$

(iv) $\mathcal{E}_{k}\left(A^{*}\right)=\overline{\mathcal{E}_{k}(A)}$

(v) $\mathcal{E}_{k}\left(a A+b I_{n}\right)=a \mathcal{E}_{k}(A)+b$ if $a, b \in \mathbb{C}$

(vi) $\Lambda_{k+1}(A) \subset \mathcal{E}_{k}(A)$, where $\Lambda_{k+1}(A)$ is the $(k+1)$-rank numerical range of $A$

Proof. Statement $(i)$ is clear from above, and $(i i)-(v)$ follow directly from Proposition 4. $a \in \mathbb{C}$ is allowed since we use all rotations $e^{i \theta} A$ to define $\mathcal{E}_{k}(A)$. By Theorem $5, \Gamma_{k}\left(e^{i \theta} A\right) \subset$ $\left\{s+i t ; s \geq \delta_{k+1}\left(e^{i \theta} A\right)\right\}$ which implies $\left\{s+i t ; s \leq \delta_{k+1}\left(e^{i \theta} A\right)\right\} \subset E_{k}\left(e^{i \theta} A\right)$. Thus $\Lambda_{k+1}(A)=$ $\bigcap_{\theta \in[0,2 \pi[} e^{-i \theta}\left\{s+i t ; s \leq \delta_{k+1}\left(e^{i \theta} A\right)\right\} \subset \bigcap_{\theta \in[0,2 \pi[} e^{-i \theta} E_{k}\left(e^{i \theta} A\right)=\mathcal{E}_{k}(A)$ which proves $(v i)$.

We now give several illustrations of $\mathcal{E}_{2}(A)$. All plots are made using 120 curves, separated by 3 degrees, that is, $\theta=2 \pi m / 120, m=0, \ldots, 119$. In Figure 1 the regions $F(A), \mathcal{E}_{1}(A)$ and $\mathcal{E}_{2}(A)$ for the Toeplitz matrix in (1) are illustrated. This matrix was used as an example in [5] to illustrate $\mathcal{E}_{1}(A)$.

On the top row of Figure 10 we show $F\left(A_{1}\right), \mathcal{E}_{1}\left(A_{1}\right)$ and $\mathcal{E}_{2}\left(A_{1}\right)$ for the matrix

$$
A_{1}=\left(\begin{array}{cccc}
14+19 i & -4-i & -55-13 i & -32+13 i \\
27+2 i & 14-25 i & 64 & 72 \\
54+i & 47-3 i & 14+44 i & -32-42 i \\
76 & 73 & 4-2 i & -11+24 i
\end{array}\right)
$$

that was used as an example in [6]. Here we observe that $\mathcal{E}_{1}\left(A_{1}\right)$ isolates one and $\mathcal{E}_{2}\left(A_{1}\right)$ two of the eigenvalues, which are marked by small boxes. 
On the middle row of Figure 10 are the corresponding regions for the $11 \times 11$ Frank matrix $A_{2}$ used in [5] to illustrate $\mathcal{E}_{1}\left(A_{2}\right)$. Frank matrices have elements $A_{i j}=0$ if $j \leq i-2, A_{i j}=n+1-i$ if $j=i-1$, and $A_{i j}=n+1-j$ if $j \geq i$. They have determinant 1 but are ill-conditioned.

On the bottom row of Figure 10 are the corresponding regions for a real random $5 \times 5$ matrix $A_{3}$ with elements between -1 and 1 , and 5 real eigenvalues, $\mathcal{E}_{1}\left(A_{3}\right)$ isolates two of them and $\mathcal{E}_{2}\left(A_{3}\right)$ three.
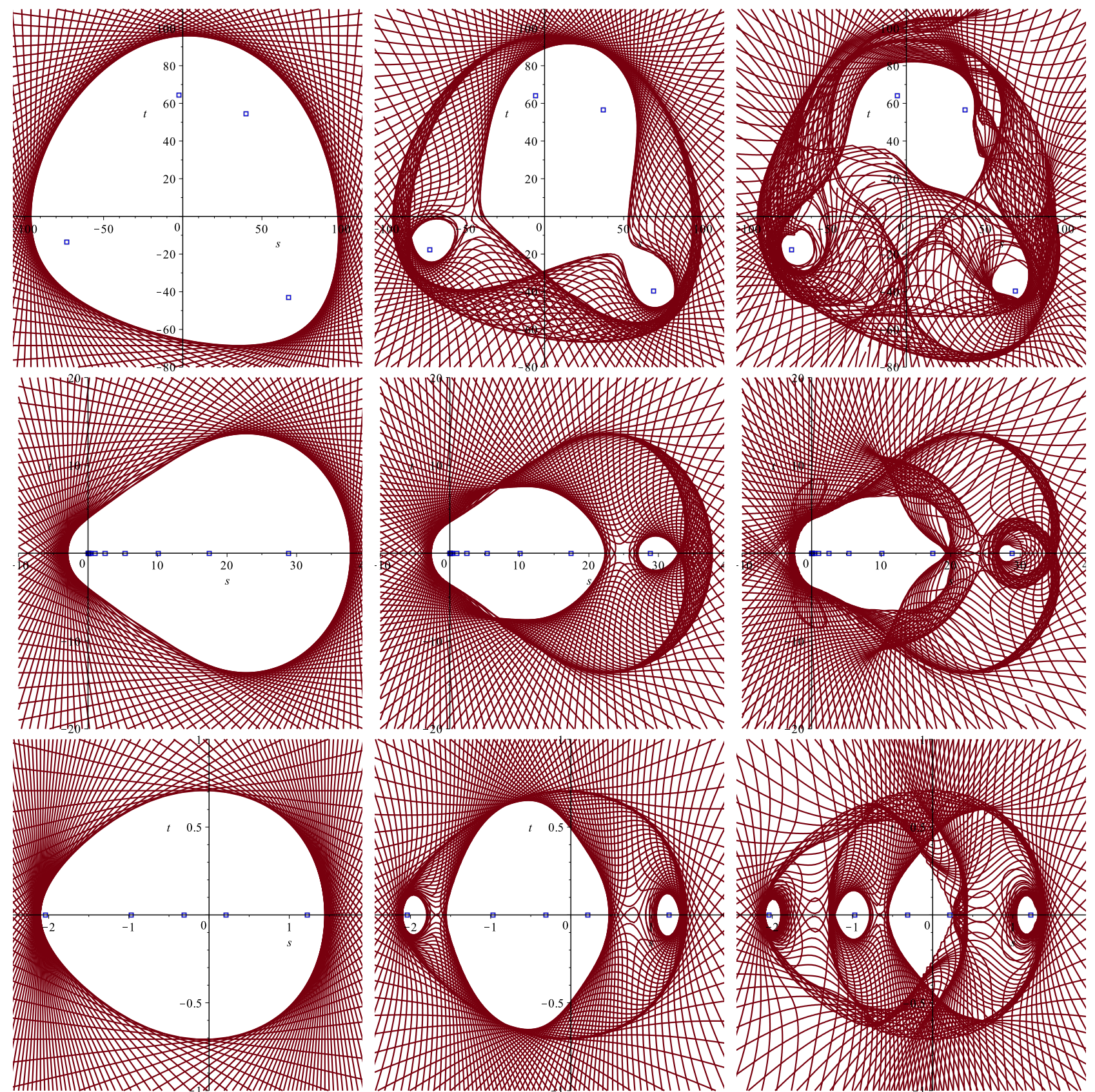

Figure 10: The numerical range $F(A)$ (left), and the regions $\mathcal{E}_{1}(A)$ (center) and $\mathcal{E}_{2}(A)$ (right) of: the matrix $A_{1}$ in (36) (top), the $11 \times 11$ Frank matrix $A_{2}$ in [5] (middle), and a real random $5 \times 5$ matrix $A_{3}$ (bottom). 
Finally, we can observe from simulations that $\mathcal{E}_{1}(A)$ can exclude regions of the complex plane not excluded by $\mathcal{E}_{2}(A), \mathcal{E}_{2}(A) \not \subset \mathcal{E}_{1}(A)$, so the single curve property shown in Figure 5 can be noticed also for the envelopes. In Figure 11 we illustrate this for a complex random $5 \times 5$ matrix $A_{4}$ with real and imaginary parts of the elements between -1 and 1 . The cubic envelope $\mathcal{E}_{1}\left(A_{4}\right)$ successfully isolates one of the eigenvalues, marked by small boxes, but not $\mathcal{E}_{2}\left(A_{4}\right)$. In the fourth subfigure we observe how the intersection $\mathcal{E}_{1}\left(A_{4}\right) \cap \mathcal{E}_{2}\left(A_{4}\right)$ of both regions determines a new smaller region for the spectrum.
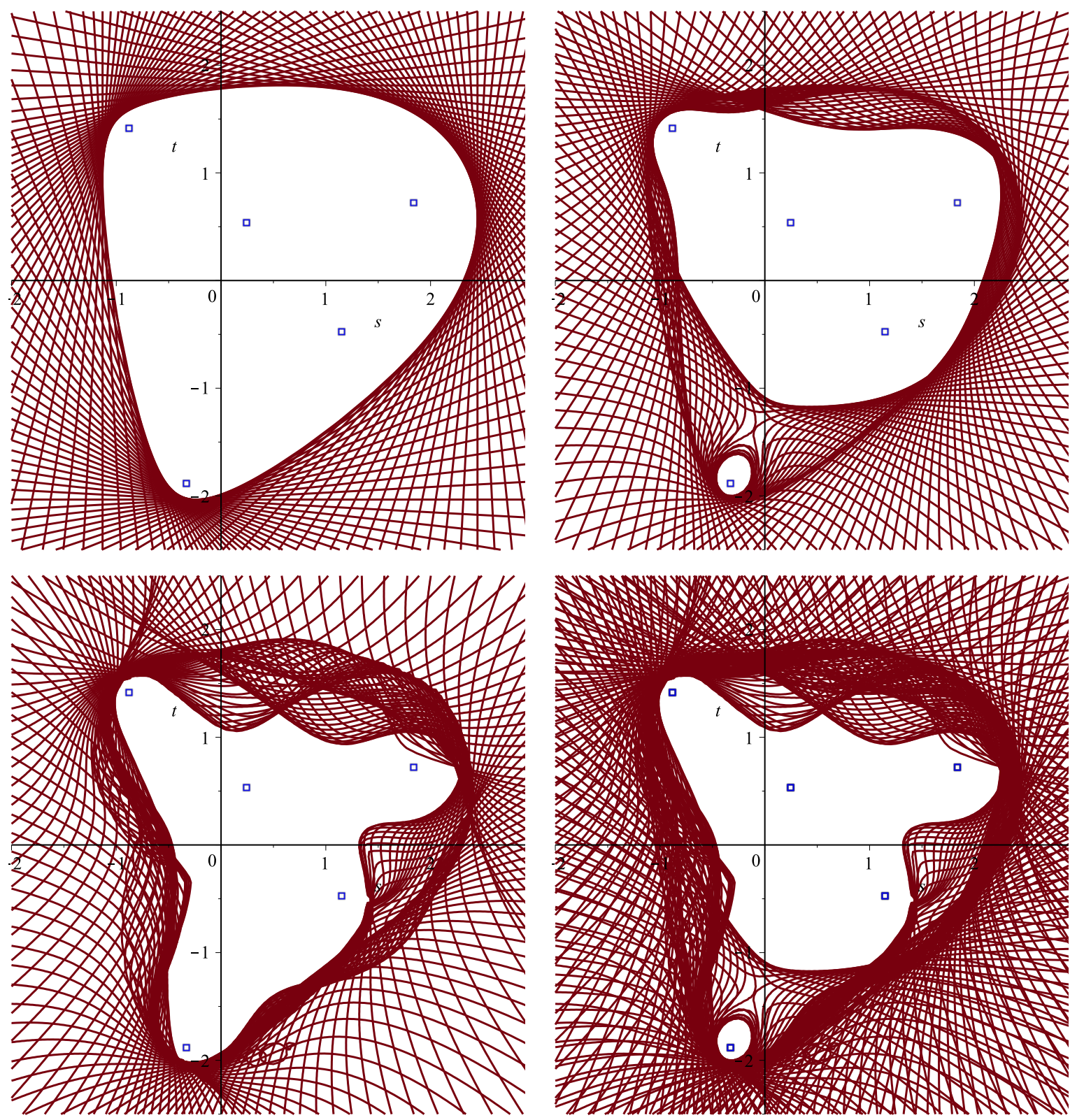

Figure 11: The numerical range $F\left(A_{4}\right)$ (top left), the regions $\mathcal{E}_{1}\left(A_{4}\right)$ (top right), $\mathcal{E}_{2}\left(A_{4}\right)$ (bottom left), and the intersection $\mathcal{E}_{1}\left(A_{4}\right) \cap \mathcal{E}_{2}\left(A_{4}\right)$ (bottom right) of a complex random $5 \times 5$ matrix $A_{4}$. 


\section{Open problems and discussion}

Adam, Psarrakos and Tsatsomeros [1, 5, 6] have proven several properties of $\Gamma_{1}(A)$ and $\mathcal{E}_{1}(A)$ that need to be studied for $k \geq 2$.

They proved that when there is a closed loop of $\Gamma_{1}(A)$, then there is precisely one simple eigenvalue inside. We have seen that we can construct matrices where $\Gamma_{k}(A)$ has a closed loop enclosing $k$ eigenvalues, or, more generally for any $1 \leq j \leq k, j$ closed loops enclosing $n_{1}, \ldots, n_{j}$ eigenvalues respectively, for any positive $n_{1}, \ldots, n_{j}$ with $j \leq n_{1}+\cdots+n_{j} \leq k$. It would be desirable to have a theorem giving a more precise description of possible topologies of $\Gamma_{k}(A)$.

The important result that $\mathcal{E}_{1}(A)$ is always compact is proved in [6], and it is likely that this should hold also for $\mathcal{E}_{k}(A)$ for $k \geq 2$. The study of normal matrices or normal eigenvalues [5, 6] should also be generalized.

We have seen that $\Gamma_{2}(A)$ is not always more restricting for the spectrum than $\Gamma_{1}(A)$ everywhere in the complex plane, and that $\mathcal{E}_{2}(A)$ is not necessarily a subset of $\mathcal{E}_{1}(A)$. These facts need to be further studied and relations between different $\Gamma_{k}(A)$ or different $\mathcal{E}_{k}(A)$ need to be explored.

The level of improvement for increasing $k$, i.e., the reduction in size of $\mathcal{E}_{k}(A)$ which depends on the separation of $\delta_{1}, \delta_{2}, \delta_{3}, \ldots$ might be possible to quantify. For large complex random matrices with most eigenvalues within some bound, the improvement will be smaller than for certain types of more structured matrices.

The computational complexity increases fast with increasing $k$, especially for the envelope $\mathcal{E}_{k}(A)$. It might be possible to find less restrictive inequalities for the spectrum, but which produce curves and regions that are easier to analyze and construct numerically.

\section{References}

[1] M Adam and M J Tsatsomeros "An eigenvalue inequality and spectrum localization for complex matrices" Electr. J. Lin. Alg. 15 (2006) 239-250

[2] R A Horn and C R Johnson Topics in Matrix Analysis Cambridge University Press (Cambridge), 1991

[3] C R Johnson "Numerical determination of the field of values of a general complex matrix" SIAM J. Numer. Anal. 15 (1978) 595-602

[4] R Mathias "Matrices with positive definite Hermitian part: Inequalities and linear systems" SIAM J. Matrix Anal. Appl. 13 (1992) 640-654

[5] P J Psarrakos and M J Tsatsomeros "An envelope for the spectrum of a matrix" Cent. Eur. J. Math. 10 (2012) 292-302

[6] P J Psarrakos and M J Tsatsomeros "On the geometry of the envelope of a matrix" Appl. Math. Comp. 244 (2014) 132-141 